\title{
Enumerating the operators of an effective field theory
}

\author{
Renato M. Fonseca $\odot^{*}$ \\ Institute of Particle and Nuclear Physics, Faculty of Mathematics and Physics, \\ Charles University, V Holešovičkách 2, 18000 Prague 8, Czech Republic
}

(Received 12 November 2019; accepted 31 January 2020; published 28 February 2020)

\begin{abstract}
Until recently little was known about the high-dimensional operators of the standard model effective field theory (SMEFT). However, in the past few years the number of these operators has been counted up to mass dimension 15 using techniques involving the Hilbert series. In this work I will show how to perform the same counting with a different method. This alternative approach makes it possible to cross-check results (it confirms the SMEFT numbers), but it also provides some more information on the operators beyond just counting their number. The considerations made here apply equally well to any other model besides SMEFT and, with this purpose in mind, they were implemented in a computer code.
\end{abstract}

DOI: 10.1103/PhysRevD.101.035040

\section{INTRODUCTION}

It is sometimes useful to consider interactions which are allowed by symmetry, even if they are not renormalizable. Seen as effective interactions, they can be used to study the effects of new, higher-energy physics in a modelindependent way.

For example, one can take the Standard Model fields and build all those operators which are invariant under gauge and Lorentz transformations, including those with a mass dimension larger than four. This construction is often called the standard model effective field theory (SMEFT), and it has been studied for a long time. The unique dimension five term in SMEFT, which violates the lepton number and may explain neutrino masses, was mentioned for the first time in [1]. This very same paper, as well as [2,3], also lists the dimension six terms which violate the baryon number (hence they can induce nucleon decay), while the remaining baryon number conserving operators with this dimension were listed in [4-6]. Nevertheless, for most purposes several of these operators can be shown to be redundant, and the authors of [7] were the first to provide a complete list of nonredundant SMEFT Lagrangian terms up to dimension six. The counting of how many parameters are contained in such terms was given in [8].

It is surprising that it took so long to fully understand these operators, given that their dimension is not particularly large: most of them have four fields or less. Currently,

\footnotetext{
*fonseca@ipnp.mff.cuni.cz
}

Published by the American Physical Society under the terms of the Creative Commons Attribution 4.0 International license. Further distribution of this work must maintain attribution to the author(s) and the published article's title, journal citation, and DOI. Funded by SCOAP. the operators of dimension seven also seem to be well understood [9-11], but beyond that there are only partial results. For example, some lepton number violating operators were presented in [12,13], and reference [14] lists field combinations which violate the lepton number in four units but without building the operators explicitly nor checking for redundancies.

However, in the past few years, there has been remarkable progress in the counting of operators, using the Hilbert series [15-18]; see also [19-23] for earlier works on this topic. This technique is not only very elegant and simple to use, but it can also be applied to an arbitrary model, with an arbitrary symmetry group (see, for example, [24-26]). Using it, the authors of [18] computed the number of SMEFT operators up to dimension 15.

Despite these advantages, the computations performed in the Hilbert series method are very different from those performed in what I will call the traditional method, which consists of simply multiplying all fields in all possible ways and retaining those combinations which are invariant under the action of all relevant symmetry groups-usually the Lorentz and gauge groups. The choice of the word traditional in this context is justified by the fact that historically the allowed Lagrangians terms have been found in this way.

There are two complications to this latter approach. One of them is also present in the Hilbert series method, and it is related to operators with derivatives: they are problematic mainly because some combinations of these operators are redundant (under some assumptions), and it would therefore be desirable to remove them. But it turns out that the ideas used to handle this issue in Hilbert series computations $[16,18]$ work equally well when adapted to the traditional method of taking all invariant fields combinations. 
For this reason, the main focus of this document will be the other complication, which arises when there are repeated fields in an operator. The simplest example in SMEFT is $L L H H$, which generates neutrino masses when $H$ acquires a vacuum expectation value. For $n$ copies of the field $L$, one might have thought that such a term is parametrized by $n^{2}$ complex numbers. However, this is not true: the coupling matrix $\kappa$ in flavor space appearing in the expression $\kappa_{i j} L_{i} L_{j} H H$ is symmetric $\left(\kappa_{i j}=\kappa_{j i}\right)$, so the term is parametrized by just $n(n+1) / 2$ complex numbers. This is purely a consequence of the quantum numbers of $L$ and $H$, as I will discuss in detail later on. It is easy to find more complicated examples, such as

$$
N^{c} N^{c} N^{c} N^{c} \text { and } Q Q Q L,
$$

where $N^{c}$ represents right-handed neutrino fields (they do not exist in SMEFT), which are gauge singlets. While the dimension of these terms (six) is still quite small, it is nontrivial to derive the number of independent couplings associated with each, assuming $n_{X}$ flavors of the field type $X=N^{c}, Q, L{ }^{1}$

Fortunately, these two complications can be handled in a systematic way. This makes it possible to count operators in models such as SMEFT, up to high mass dimensions, using the traditional approach mentioned earlier. In fact, besides the number of operators, it is also possible to extract the symmetry of coupling tensors (such as $\kappa$ above) under flavor index permutations; in some cases, this is a piece of extra information which cannot be inferred from the number of operators.

It is also noteworthy that the traditional method described in this work seems to be computationally competitive with the Hilbert series approach. The authors of [18] provided the number of each type of SMEFT operator up to dimension 12 plus the total number of operators with dimensions $d=13,14$, and 15 . Up to now, these results had only been cross-checked by other means up to $d=8$. However, with the method explained in this work it was possible to count all types of SMEFT operators up to dimension 15 in a couple of hours, using a standard laptop (all numbers given in [18] were reproduced). Existing Mathematica code $[27,28]$ was modified to make such calculations possible for any model, and such code is publicly available at the web address renatofonseca.net/ sym2int.php.

The remainder of the text is structured as follows:

(i) Section II describes the notation and the conventions adopted in this work.

(ii) Section III is devoted to the problem of repeated fields in interactions, suggesting a way of systematically dealing with these cases.

\footnotetext{
${ }^{1}$ The answer is $\frac{n_{N^{c}}^{2}}{12}\left(n_{N^{c}}^{2}-1\right)$ and $\frac{n_{Q} n_{L}}{3}\left(2 n_{Q}^{2}+1\right)$.
}

(iii) Section IV discusses the problem with derivatives, and how they can be handled by using and adapting the solutions proposed in $[16,18]$.

(iv) Section V contains a discussion of several topics related to the counting of operators of an effective field theory. The ideas mentioned in Secs. III and IV were implemented in a computer code, and some of the results obtained with it are presented here and in Appendix.

(v) A summary is available at the end.

\section{NOTATION AND CONVENTIONS}

The method discussed in this work can be applied to any effective field theory; nevertheless, SMEFT will often serve as an example. Its Lagrangian is invariant under the $S U(3)_{C} \times S U(2)_{L} \times U(1)_{Y}$ gauge group and the restricted Lorentz group. ${ }^{2}$ For all practical purposes the algebra of this latter group can be taken to be the same as the one of $S U(2)_{l} \times S U(2)_{r}$ so its representations are given by pairs of non-negative half-integers $\left(j_{l}, j_{r}\right)$. The only caveat to keep in mind is that complex conjugation flips these numbers: $\left(j_{l}, j_{r}\right)^{*}=\left(j_{r}, j_{l}\right)$. In particular, the representations $(0,0),\left(\frac{1}{2}, 0\right),\left(0, \frac{1}{2}\right)$, and $\left(\frac{1}{2}, \frac{1}{2}\right)$ correspond to a scalar, a left-handed Weyl spinor, a right-handed Weyl spinor, and a 4 -vector, in this order. A field strength tensor $\mathcal{F}_{\mu \nu}$ transforms under the Lorentz group in the same way as the antisymmetric part of the product of two 4-vectors, i.e.,

$$
\left[\left(\frac{1}{2}, \frac{1}{2}\right) \times\left(\frac{1}{2}, \frac{1}{2}\right)\right]_{A}=(1,0)+(0,1) .
$$

Note that $\mathcal{F}_{\mu \nu}$ is a real field so the three components transforming as $(0,1)$ are the complex conjugate of those transforming as $(1,0)$. I will refer to the part transforming as $(1,0)$ simply with the letter $F$ and the other part as $F^{*}$ (so $\mathcal{F}_{\mu \nu}=F+F^{*}$ ). A subscript might be added to $F$ to identify with which of the gauge factor groups it is associated.

All SMEFT operators are combinations of the fields shown in Table I, their conjugates, and their derivatives (which need to be covariant). There are three copies (or flavors) of each fermion field, but we may just treat this number as a variable $n$.

Finally, concerning conventions, it is worth noting that sometimes the word operator is used in reference to different things:

(1) It might refer to the different gauge and Lorentz invariant contraction of fields, with the flavor indices expanded. In that case the lepton Yukawa interactions $L_{i} e_{j}^{c} H^{*}$ correspond to 9 complex operators,

\footnotetext{
${ }^{2}$ The word restricted here refers to the fact that time-reversal $(T)$ and space-inversion $(P)$ transformations are not included. This group is usually denoted by $\mathrm{SO}^{+}(1,3)$.
} 
TABLE I. SMEFT field content. All operators are built from them, their conjugates, and their derivatives. The number of fermion flavors $n$, which is 3 , will often be left unspecified.

\begin{tabular}{lccccc}
\hline \hline & $S U(3)_{C}$ & $S U(2)_{L}$ & $U(1)_{Y}$ & $S U(2)_{l} \times S U(2)_{r}$ & \# flavors \\
\hline$Q$ & $\mathbf{3}$ & $\mathbf{2}$ & $\frac{1}{6}$ & $\left(\frac{1}{2}, 0\right)$ & $n$ \\
$u^{c}$ & $\overline{\mathbf{3}}$ & $\mathbf{1}$ & $-\frac{2}{3}$ & $\left(\frac{1}{2}, 0\right)$ & $n$ \\
$d^{c}$ & $\overline{\mathbf{3}}$ & $\mathbf{1}$ & $\frac{1}{3}$ & $\left(\frac{1}{2}, 0\right)$ & $n$ \\
$L$ & $\mathbf{1}$ & $\mathbf{2}$ & $-\frac{1}{2}$ & $\left(\frac{1}{2}, 0\right)$ & $n$ \\
$e^{c}$ & $\mathbf{1}$ & $\mathbf{1}$ & 1 & $\left(\frac{1}{2}, 0\right)$ & $n$ \\
$H$ & $\mathbf{1}$ & $\mathbf{2}$ & $\frac{1}{2}$ & $(0,0)$ & 1 \\
$F_{G}$ & $\mathbf{8}$ & $\mathbf{1}$ & 0 & $(1,0)$ & 1 \\
$F_{W}$ & $\mathbf{1}$ & $\mathbf{3}$ & 0 & $(1,0)$ & 1 \\
$F_{B}$ & $\mathbf{1}$ & $\mathbf{1}$ & 0 & $(1,0)$ & 1 \\
\hline \hline
\end{tabular}

or 18 real ones. That is the meaning I will use for the word operator (which might be real or complex). With this understanding, there are 3045 dimension six real operators in SMEFT (546 of which violate the baryon number) $[8,18]$.

(2) An alternative view is to see some groups of operators such as the lepton Yukawa interactions $L_{i} e_{j}^{c} H^{*}$ as a single structure which I will call a (Lagrangian) term; they can be thought of as operators with the flavor indices unexpanded. With this terminology, SMEFT can be written with just 84 real terms of mass dimension six [7].

(3) The most general interaction of some combinations of fields cannot be written in the Lagrangian as a single term. In other words, a single coupling tensor $\kappa_{i j \ldots}$. with indices $i j \ldots$ contracted with the field flavors is not enough. That is what happens, for example, with the fields $Q^{*} L^{*} Q L$ : a minimum of two term $\kappa^{(\alpha)}{ }_{i j k l}\left(Q_{i}^{*} L_{j}^{*} Q_{k} L_{l}\right)_{(\alpha)}, \alpha=1,2$ are needed.

We may refer to each collection of terms associated with a common product of fields as a type of operator. ${ }^{3}$ There are 72 real types of operators with dimension six in SMEFT.

\section{OPERATORS WITH REPEATED FIELDS}

\section{A. The problem}

If there are no derivatives nor repeated fields, the counting of operators of a certain type $\chi^{(1)} \chi^{(2)} \cdots \chi^{(m)}$ is straightforward:

(i) Based on the quantum numbers of the fields, we may calculate the number $t$ of independent gauge and Lorentz invariant contractions of their components.

\footnotetext{
${ }^{3}$ So each operator type is defined by the number of occurrences $m_{i}$ of the various kinds of field $\chi_{i}$ in the model $\left(=Q, u^{c}, d^{c}, \ldots\right.$ in SMEFT). For these purposes, a derivative may be considered as a field, so we should just count them.
}

Each yields one term. These contractions can be found systematically with the method of weights $[29,30]$ or, in the case of special unitary groups, with the tensor method (see for instance Chap. 4 of [31]).

(ii) Each term is associated with $\prod_{i=1}^{m} n_{i}$ operators, where $n_{i}$ is the number of flavors of the field $\chi^{(i)}$.

(iii) The number quoted above corresponds to real operators if conjugating the set of fields $\left\{\chi^{(i)}\right\}$ yields back this very same set, i.e., $\left\{\chi^{(i)}\right\}^{*}=\left\{\chi^{(i)}\right\}$; otherwise there are $\prod_{i=1}^{m} n_{i}$ complex operators in each term (which is the same as saying that there are $2 \prod_{i=1}^{m} n_{i}$ real operators).

So, in the absence of derivatives and repeated fields, there are $c t \prod n_{i}$ real operators of type $\chi^{(1)} \chi^{(2)} \cdots \chi^{(m)}$, where $c=1$ if $\left\{\chi^{(i)}\right\}^{*}=\left\{\chi^{(i)}\right\}$ and $c=2$ otherwise. For example, there are $2 n^{4}=162$ (for $n=3$ ) real operators of type $Q^{*} L^{*} Q L{ }^{4}$

The above is straightforward to compute. Nevertheless, repeated fields complicate this analysis significantly (the problem with derivatives will be discussed in the next section).

Let us start by taking a close look at the type of operator $L L H H$, which is not associated with $n^{2}$ complex operators, but rather with $n(n+1) / 2$ as pointed out earlier. Given that $L$ and $H$ are both doublets of $S U(2)_{L}$, there are two possible contractions: $(L L)_{\mathbf{1}}(H H)_{\mathbf{1}}$ and $(L L)_{\mathbf{3}}(H H)_{3}$. In one of them, the two $L$ 's are contracted together as a singlet of $S U(2)_{L}$, and the same happens with the Higgs fields. The other possibility is for the $L$ 's and $H$ 's to contract as triplets. Crucially, the singlet contraction of two doublets is antisymmetric, while the triplet contraction is symmetric. Since there is only one copy of the Higgs field, we retain only the latter. If we now introduce flavor indices and a parameter tensor $\kappa$, we obtain the term

$$
\kappa_{i j}\left(L_{i} L_{j}\right)_{\mathbf{3}}(H H)_{\mathbf{3}} .
$$

This is the same as $\kappa_{i j}\left(L_{j} L_{i}\right)_{\mathbf{3}}(H H)_{\mathbf{3}}$ so, renaming the dummy indices $i$ and $j$, we can also write it as $\kappa_{j i}\left(L_{i} L_{j}\right)_{\mathbf{3}}(H H)_{\mathbf{3}}$. We then conclude that only the symmetric part of $\kappa$ is relevant, ${ }^{5}$

$$
\kappa_{i j}=\kappa_{j i}
$$

Such a matrix has $n(n+1) / 2$ complex degrees of freedom.

Now let us go beyond SMEFT for a moment in order to consider what would happen to the type of operator $\mathrm{LLHH}$

\footnotetext{
${ }^{4}$ The list of fields is self-conjugate, $\left\{Q^{*}, L^{*}, Q, L\right\}^{*}=$ $\left\{Q^{*}, L^{*}, Q, L\right\}$, so $c=1$. On the other hand, there are two ways of making the $S U(2)_{L}$ contractions of the fields, so $t=2$.

${ }^{5}$ One could add an antisymmetric part to this matrix $\kappa$, but it would not affect the Lagrangian. So it is pointless to do so.
} 
if there were multiple copies of the Higgs field. For that purpose we may add flavor indices to $H$, noting that now the contraction $(L L)_{\mathbf{1}}(H H)_{\mathbf{1}}$ is not null so we can have two terms:

$$
\kappa_{i j k l}^{(1)}\left(L_{i} L_{j}\right)_{\mathbf{1}}\left(H_{k} H_{l}\right)_{\mathbf{1}}+\kappa_{i j k l}^{(3)}\left(L_{i} L_{j}\right)_{\mathbf{3}}\left(H_{k} H_{l}\right)_{\mathbf{3}} .
$$

It should be clear from the discussion so far that the tensors $\kappa^{(1)}$ and $\kappa^{(2)}$ have symmetries:

$$
\begin{gathered}
\kappa_{i j k l}^{(1)}=-\kappa_{j i k l}^{(1)}=-\kappa_{i j l k}^{(1)}, \\
\kappa_{i j k l}^{(3)}=\kappa_{j i k l}^{(3)}=\kappa_{i j l k}^{(3)} .
\end{gathered}
$$

As a consequence, $\kappa^{(1)}$ and $\kappa^{(3)}$ contain

$$
[n(n-1) / 2]\left[n_{H}\left(n_{H}-1\right) / 2\right]
$$

and

$$
[n(n+1) / 2]\left[n_{H}\left(n_{H}+1\right) / 2\right]
$$

complex parameters, respectively $\left(n_{H}\right.$ being the number of Higgs copies). But do we need two terms as in expression (5)? The answer is no; there is no such need. Take a single term $\mathcal{O}$ formed from a linear combination of $\mathcal{O}^{(1)} \equiv$ $(L L)_{\mathbf{1}}(H H)_{\mathbf{1}}$ and $\mathcal{O}^{(3)} \equiv(L L)_{\mathbf{3}}(H H)_{\mathbf{3}}$ :

$$
\mathcal{O}=\alpha_{1} \mathcal{O}^{(1)}+\alpha_{3} \mathcal{O}^{(3)} \quad\left(\alpha_{1}, \alpha_{3} \neq 0\right) .
$$

We can recover $\mathcal{O}^{(1)}$ and $\mathcal{O}^{(3)}$ from $\mathcal{O}$ by (anti)symmetrizing the flavor indices, $\mathcal{O}_{i j k l}^{(1)}=\alpha_{1}^{-1} \mathcal{O}_{[i j][k l]}$ and $\mathcal{O}_{i j k l}^{(3)}=\alpha_{3}^{-1} \mathcal{O}_{(i j)(k l)}$, and therefore we can write

$$
\kappa_{i j k l} \mathcal{O}_{i j k l}
$$

instead of expression (5). Nevertheless, the tensor $\kappa$ is not fully generic, as it contains only $n n_{H}\left(n n_{H}+1\right) / 2$ independent complex parameters (the sum of the number of parameters in $\kappa^{(1)}$ and $\left.\kappa^{(3)}\right)$. In fact, we may write

$$
\kappa=\alpha_{1}^{-1} \kappa^{(1)}+\alpha_{3}^{-1} \kappa^{(3)}
$$

or, alternatively, $\kappa$ can be described as the most general tensor with the symmetry $\kappa_{i j k l}=\kappa_{j i l k}$.

Returning to SMEFT, an identical situation happens with the type of operator $L^{*} L^{*} L L$ : four $S U(2)_{L}$ doublets can be contracted in two different ways [6], but only one term is required [7], and it is associated with a total of $n^{2}\left(n^{2}+1\right) / 2=45$ complex operators [8] (this last expression is the same as the one presented before in relation to $L L H H$, for the special case where $n_{H}=n$ ).
These examples highlight the following points:

(i) The symmetry of the coupling tensors $\kappa$ is determined by the quantum numbers of the fields in each term.

(ii) From the symmetry of the coupling tensors we may derive the number of independent operators.

(iii) Unlike the number of independent operators, the number of terms is an ambiguous quantity since it is possible to merge multiple terms into a single one. To get around this ambiguity, we may always focus on writing a Lagrangian with a minimal number of terms.

(iv) Merging terms might seem convenient, but there is a drawback to doing so: it may become more complicated to understand the structure of the associated coupling tensor $\kappa$.

In order to derive the symmetry of the coupling tensors under permutations of indices, we may consult tables such as in [29] which list the permutation symmetry of products of representations. For example, in the case of $S U(2)$ it is well known that

$$
\mathbf{2} \times \mathbf{2}=\mathbf{1}_{A}+\mathbf{3}_{S},
$$

with the subscripts indicating that each component in the product is either symmetric (S) or antisymmetric (A) under a switch of the two doublets. ${ }^{6}$ However, such tables might not be enough: consider, for example, the product of four scalar $S U(2)$ doublets. There are two invariant contractions because the product of four doublets contains two singlets:

$$
2 \times 2 \times 2 \times 2=1+1+\cdots .
$$

Are these singlets symmetric or antisymmetric? Mathematically, the answer is clear but it might be somewhat confusing if one is unaware of this type of complications: the singlets are neither (completely) symmetric nor antisymmetric. Let us go back to Eq. (13); as far as subscripts are concerned, all we need to know is what happens to each summand in the right-hand side under the permutation of the two doublets. The permutation of $m$ objects forms a discrete group of size $m$ ! usually denoted by $S_{m}$, and in the particular case of $S_{2}$ everything is very simple. There are two elements in the group, the identity $e$ (do nothing) and $a$ (transpose the two objects), with $a^{2}=e$. There are also only two irreducible representations, which are both one-dimensional: the trivial/symmetric one (S) under which $x \mapsto x$, and the alternating/antisymmetric representation (A) under which $x \mapsto(-1)^{\pi} x$, where $(-1)^{\pi}=+1$ if $\pi=e$ and $=-1$ if $\pi=a$. In other words,

\footnotetext{
${ }^{6}$ To be explicit, it is well known that if $\left(2_{1}, 2_{2}\right)^{T}$ and $\left(2_{1}^{\prime}, 2_{2}^{\prime}\right)^{T}$ are two doublets, then the combination $2_{1} 2_{2}^{\prime}-22_{2}^{\prime}$ is $S U(2)$ invariant, while $\left(2_{1} 2_{1}^{\prime}, \frac{1}{\sqrt{2}} 2_{1} 2_{2}^{\prime}+\frac{1}{\sqrt{2}} 2_{2} 2_{1}^{\prime}, 2_{2} 2_{2}^{\prime}\right)^{T}$ transforms as a triplet. Switching the two doublets flips the sign of the first expression, while the triplet remains unchanged.
} 
the action of the group $S_{2}$ can always be translated into signs \pm 1 : in Eq. (13) the singlet 1 transforms under $S_{2}$ as the alternating representation, while the components of the triplet 3 transform trivially.

In the case of Eq. (14) we must find out what happens to its right-hand side under arbitrary permutations of the four doublets, so we must deal with the irreducible representations of the $S_{4}$ group. It turns out that the singlets in Eq. (14) transform as an irreducible two-dimensional representation of $S_{4}$. To better grasp the significance of this statement, let us see these two $S U(2)$-invariant contractions of four doublets explicitly. We can write them as follows:

$$
\begin{aligned}
& c^{(1)}=\epsilon_{i j} \epsilon_{k l} 2_{i} 2_{j}^{\prime} 2_{k}^{\prime \prime} 2_{l}^{\prime \prime \prime}, \\
& c^{(2)}=\epsilon_{i k} \epsilon_{j l} 2_{i} 2_{j}^{\prime} 2_{k}^{\prime \prime} 2_{l}^{\prime \prime \prime},
\end{aligned}
$$

where the subscripts take the value 1 or 2 (these are doublet indices unrelated to flavor) and $\epsilon$ is the Levi-Civita tensor. Note than even though there are $m$ ! permutations which can be performed on $m$ objects $x_{i}$, all of them can be obtained through successive applications of only two of them: $x_{1} \rightarrow$ $x_{2} \rightarrow x_{1}$ and $x_{1} \rightarrow x_{2} \rightarrow x_{3} \rightarrow \cdots \rightarrow x_{m} \rightarrow x_{1}$. So, let us consider in the following what happens to $c^{(1)}$ and $c^{(2)}$ under the changes $\mathbf{2} \leftrightarrow \mathbf{2}^{\prime}$ and $\mathbf{2} \rightarrow \mathbf{2}^{\prime} \rightarrow \mathbf{2}^{\prime \prime} \rightarrow \mathbf{2}^{\prime \prime \prime} \rightarrow \mathbf{2}$. It is rather easy to see that

$$
\begin{gathered}
\left(\begin{array}{l}
c^{(1)} \\
c^{(2)}
\end{array}\right)_{\mathbf{2} \leftrightarrow \mathbf{2}^{\prime}}=\left(\begin{array}{ll}
-1 & 0 \\
-1 & 1
\end{array}\right) \cdot\left(\begin{array}{l}
c^{(1)} \\
c^{(2)}
\end{array}\right), \\
\left(\begin{array}{l}
c^{(1)} \\
c^{(2)}
\end{array}\right)_{{\mathbf{2} \rightarrow \mathbf{2}^{\prime} \rightarrow \mathbf{2}^{\prime \prime} \rightarrow \mathbf{2}^{\prime \prime \prime} \rightarrow \mathbf{2}}}=\left(\begin{array}{ll}
1 & -1 \\
0 & -1
\end{array}\right) \cdot\left(\begin{array}{l}
c^{(1)} \\
c^{(2)}
\end{array}\right) .
\end{gathered}
$$

No change of basis $c_{\text {new }}^{(\alpha)}=B_{\alpha \beta} c^{(\beta)}$ will simultaneously diagonalize the two matrices above, so $c^{(1),(2)}$ form an irreducible two-dimensional representation of the permutation group $S_{4}$. As a consequence, in this case the effect of permuting the doublets cannot be reduced to a simple matter of signs \pm 1 .

We now introduce flavor, so there will be two sets of indices: the $g_{i}$ will stand for group indices, while flavor indices will be called $f_{i}$. For scalar doublets $\phi$ with multiple flavors we should write down two terms:

$$
\left(\kappa_{f_{1} f_{2} f_{3} f_{4}}^{(1)} c_{g_{1} g_{2} g_{3} g_{4}}^{(1)}+\kappa_{f_{1} f_{2} f_{3} f_{4}}^{(2)} c_{g_{1} g_{2} g_{3} g_{4}}^{(2)}\right) \phi_{g_{1}}^{f_{1}} \phi_{g_{2}}^{f_{2}} \phi_{g_{3}}^{f_{3}} \phi_{g_{4}}^{f_{4}},
$$

where $c_{g_{1} g_{2} g_{3} g_{4}}^{(1),(2)}$ represent the tensors in front of the doublets in Eqs. (15) and (16). As for $\kappa^{(1)}$ and $\kappa^{(2)}$, they are tensors containing free parameters, and there is some symmetry associated with them, which is yet to be determined.

Instead of writing down all the indices in Eq. (19) explicitly, we may use the shorthand notation

$$
\kappa_{\{f\}}^{(\alpha)} c_{\{g\}}^{(\alpha)} \phi_{\{g\}}^{\{f\}},
$$

where repeated indices are summed over. We know two things:

(1) $\phi_{\{g\}}^{\{f\}}$ is symmetric under equal permutation of the $f$ and $g$ indices, i.e., $\phi_{\pi\{g\}}^{\pi\{f\}}=\phi_{\{g\}}^{\{f\}}$ for any permutation $\pi$;

(2) $c_{\pi\{g\}}^{(\alpha)}=P\left(\pi^{-1}\right)_{\alpha \beta} c_{\{g\}}^{(\beta)}$, where the two-by-two matrices $P\left(\pi^{-1}\right)$ can be obtained, for any $\pi$, from the product of the two matrices in Eqs. (17) and (18). ${ }^{7}$

It follows that

$\kappa_{\{f\}}^{(\alpha)} c_{\{g\}}^{(\alpha)} \phi_{\{g\}}^{\{f\}}=\kappa_{\pi\{f\}}^{(\alpha)} c_{\pi\{g\}}^{(\alpha)} \phi_{\pi\{g\}}^{\pi\{f\}}=\kappa_{\pi\{f\}}^{(\alpha)} P\left(\pi^{-1}\right)_{\alpha \beta} c_{\{g\}}^{(\beta)} \phi_{\{g\}}^{\{f\}}$,

so

$$
\begin{aligned}
\kappa_{\pi\{f\}}^{(\alpha)} P\left(\pi^{-1}\right)_{\alpha \beta} & =\kappa_{\{f\}}^{(\beta)} \quad \text { or equivalently } \\
\kappa_{\pi\{f\}}^{(\alpha)} & =\left[P(\pi)^{T}\right]_{\alpha \beta} \kappa_{\{f\}}^{(\beta)} .
\end{aligned}
$$

To be explicit,

$$
\begin{aligned}
& \left(\begin{array}{c}
\kappa_{f_{2} f_{1} f_{3} f_{4}}^{(1)} \\
\kappa_{f_{2} f_{1} f_{3} f_{4}}^{(2)}
\end{array}\right)=\left(\begin{array}{cc}
-1 & -1 \\
0 & 1
\end{array}\right) \cdot\left(\begin{array}{l}
\kappa_{f_{1} f_{2} f_{3} f_{4}}^{(1)} \\
\kappa_{f_{1} f_{2} f_{3} f_{4}}^{(2)}
\end{array}\right), \\
& \left(\begin{array}{c}
\kappa_{f_{2} f_{3} f_{4} f_{1}}^{(1)} \\
\kappa_{f_{2} f_{3} f_{4} f_{1}}^{(2)}
\end{array}\right)=\left(\begin{array}{cc}
1 & 0 \\
-1 & -1
\end{array}\right) \cdot\left(\begin{array}{l}
\kappa_{f_{1} f_{2} f_{3} f_{4}}^{(1)} \\
\kappa_{f_{1} f_{2} f_{3} f_{4}}^{(2)}
\end{array}\right) .
\end{aligned}
$$

These are the only constraints on the parameter tensors $\kappa^{(1)}$ and $\kappa^{(2)}$, so these objects can be seen as the most general tensors which satisfy the above equations. It is not very useful for our purposes to find their exact form, which is basis dependent in any case $\left(\kappa^{(1)}\right.$ and $\kappa^{(2)}$ would change under a redefinition $\left.c_{\text {new }}^{(\alpha)}=B_{\alpha \beta} c^{(\beta)}\right)$. However, just as with the $L L H H$ type of operator, it would be interesting to know how many operators there are in expression (19) or, in other words, how many independent entries there are in the $\kappa^{(\alpha)}$ tensors. The answer, which is $n_{\phi}^{2}\left(n_{\phi}^{2}-1\right) / 12$ for $n_{\phi}$ flavors of $\phi$, depends only on the fact that the $c^{(\alpha)}$ (and consequently the $\kappa^{(\alpha)}$ as well) transform under the twodimensional representation of $S_{4}$. We shall discuss below how to compute these numbers.

\footnotetext{
${ }^{7}$ The inverse permutation of $\pi, \pi^{-1}\left(\pi^{-1} \circ \pi=i d\right)$, appears here rather than $\pi$ itself for the following reason, which is not important for the present discussion. Strictly speaking, for each $\alpha$ the numbers $c_{\{g\}}^{(\alpha)}$ are not a tensor but rather the components of a tensor in some basis $\left|e_{g_{1}} e_{g_{2}} \cdots e_{g_{m}}\right\rangle \equiv\left|e_{\{g\}}\right\rangle$. In other words, the tensors are $C^{(\alpha)}=c_{\{g\}}^{(\alpha)}\left|e_{\{g\}}\right\rangle$. Under a permutation $\pi$, the basis of the tensors changes, $\left|e_{\{g\}}\right\rangle \rightarrow \pi\left(\left|e_{\{g\}}\right\rangle\right)=\left|e_{\pi\{g\}}\right\rangle$, so $\pi\left(C^{(\alpha)}\right)=$ $c_{\{g\}}^{(\alpha)}\left|e_{\pi\{g\}}\right\rangle=c_{\pi^{-1}\{g\}}^{(\alpha)}\left|e_{\{g\}}\right\rangle$. However, we know that this result must be a linear combination of the $C^{(\alpha)}, P(\pi)_{\alpha \beta} C^{(\beta)}$, and therefore we conclude that $c_{\pi\{g\}}^{(\alpha)}=P\left(\pi^{-1}\right)_{\alpha \beta} c_{\{g\}}^{(\beta)}$.
} 
There is one last remark to be made about this example. It might have seemed that we need to write down two terms as shown in expression (19), but it is easy to see that they can be merged into a single one. That is because we may write one of the $c^{(\alpha)}$ as a function of the other, with the indices permuted. In particular, from Eqs. (17) and (18) it can be inferred that $c_{g_{1} g_{2} g_{3} g_{4}}^{(2)}=c_{g_{1} g_{3} g_{2} g_{4}}^{(1)}$ or, in a shorthand notation, $c_{\pi_{23}\{g\}}^{(2)}=c_{\{g\}}^{(1)}$. Using the fact that $\phi_{\pi\{g\}}^{\pi\{f\}}=\phi_{\{g\}}^{\{f\}}$, we obtain that

$$
\begin{aligned}
\left(\kappa_{\{f\}}^{(1)} c_{\{g\}}^{(1)}+\kappa_{\{f\}}^{(2)} c_{\{g\}}^{(2)}\right) \phi_{\{g\}}^{\{f\}} & =\left(\kappa_{\{f\}}^{(1)}+\kappa_{\pi_{23}\{f\}}^{(2)}\right) c_{\{g\}}^{(1)} \phi_{\{g\}}^{\{f\}} \\
& \equiv \kappa_{\{f\}}^{\text {merged }} c_{\{g\}}^{(1)} \phi_{\{g\}}^{\{f\}} .
\end{aligned}
$$

Note that $\kappa^{\text {merged }}$ is not a completely generic four-index tensor since it has only $n_{\phi}^{2}\left(n_{\phi}^{2}-1\right) / 12$ independent entries, rather than $n_{\phi}^{4}$.

\section{B. The permutation group of $m$ objects}

These examples highlight the fact that in order to list operators and terms in the Lagrangian, it is important to have an understanding of the permutation group $S_{m}$. As such, I will briefly review here some of its properties.

The irreducible representations of $S_{m}$ can be labeled by the different ways in which the number $m$ can be partitioned. For example,

$4=3+1=2+2=2+1+1=1+1+1+1$,

so there are five partitions $\{4\},\{3,1\},\{2,2\},\{2,1,1\}$, and $\{1,1,1,1\}$, and these can be identified with the five irreducible representations of $S_{4}$. It is common to depict the partition $\lambda=\left\{\lambda_{1}, \lambda_{2}, \ldots\right\}$ of $m$ (symbolically $\lambda \vdash m$ ) with $\lambda_{1}$ boxes in a row, followed by $\lambda_{2}$ boxes in a second row, and so on, such that $\sum_{i} \lambda_{i}=m$ and $\lambda_{i+1} \leq \lambda_{i}$. These are called Young diagrams. For example,

$$
\{4\}=\square \square \square \quad\{3,1\}=\square \square \quad\{2,2\}=\square \quad\{2,1,1\}=\square \quad\{1,1,1,1\}=\square .
$$

The product of $S_{m}$ representations can easily and efficiently be decomposed in irreducible parts as in this example:

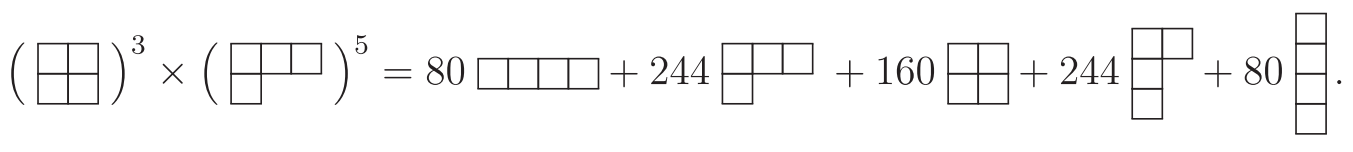

To perform such decomposition, for $S_{n}$ or any other finite group, it suffices to know the group's character table. ${ }^{8}$

There are two other important properties of the irreducible representations $\lambda$ of $S_{m}$ which are worth mentioning. First, the dimension of $\lambda$ can be calculated with the famous Hook length formula [34],

$$
d(\lambda)=\frac{m !}{\prod_{u} h(u)},
$$

where $u$ represents each box of the Young diagram of $\lambda$ and $h(u)$ is equal to the number of boxes to the right of $u$ plus the number of boxes below $u$ plus 1 :

\footnotetext{
${ }^{8}$ Readers interested in knowing more about this are referred to group theory textbooks (for instance [32]). It is worth noting that specific techniques can be applied to the $S_{n}$ family of groups (see, for example, [33]). In any case, one can also use precalculated character tables or readily available computer codes, such as GROUPMATH's function SNCLASSCHARACTER[LAMBDA, MU] which calculates the character of the class MU in the irreducible representation LAMBDA.
}

$$
h(u): \begin{array}{|l|l|l|}
\hline 4 & 2 & 1 \\
\hline 1 &
\end{array} \Rightarrow d(\{3,1\})=\frac{4 !}{4.2 .1 .1}=3 .
$$

The second important property of a partition $\lambda$ is the following. A semistandard Young tableaux with shape $\lambda$ is obtained by filling the Young diagram of $\lambda$ with natural numbers up to some limit $n$ (possibly repeating these numbers) in such a way that the numbers increase along each column, and they do not decrease along rows. For example, in the case of $\lambda=\{2,2\}$ and $n=3$ there are six possibilities:

\begin{tabular}{|l|l|}
\hline 1 & 1 \\
\hline 2 & 2 \\
\hline
\end{tabular}, \begin{tabular}{|l|l|}
\hline 1 & 1 \\
\hline 2 & 3 \\
\hline
\end{tabular}, \begin{tabular}{|l|l|}
\hline 1 & 1 \\
\hline 3 & 3 \\
\hline
\end{tabular}, \begin{tabular}{|l|l|}
\hline 1 & 2 \\
\hline 2 & 3 \\
\hline
\end{tabular}, \begin{tabular}{|l|l|}
\hline 1 & 2 \\
\hline 3 & 3 \\
\hline
\end{tabular}, \begin{tabular}{|l|l|}
\hline 2 & 2 \\
\hline 3 & 3 \\
\hline
\end{tabular}

The number of semistandard Young tableaux with a shape $\lambda$ using numbers up to $n$ can be calculated with the Hook content formula (see [35]), 


$$
\mathcal{S}(\lambda, n)=\prod_{u} \frac{n+c(u)}{h(u)},
$$

where $u$ represent each box of the diagram, $h(u)$ was mentioned earlier, and $c(u)$ is equal to the column number minus the row number of $u$ :

$$
c(u): \begin{array}{c|c|c|}
0 & 1 & 2 \\
\hline-1 & \multicolumn{2}{|c}{.} \\
\hline
\end{array}
$$

As an example, notice that $\{2,2\}$ is the two-dimensional representation of $S_{4}, d(\{2,2\})=2$, and furthermore $\mathcal{S}\left(\{2,2\}, n_{\phi}\right)=n_{\phi}^{2}\left(n_{\phi}^{2}-1\right) / 12$ using formula (32). As we have seen, this last expression corresponds to the number of quartic operators which can be build with $n_{\phi}$ scalars $\phi$ transforming as doublets of $S U(2)$.

\section{Permuting the indices of tensors}

Consider now a tensor $T_{i_{1} i_{2} \cdots i_{m}}$ where each index goes from 1 to some numbers $n$. It is well known to mathematicians that the $n^{m}$ components can either be referenced with the $m$ indices $\left(i_{1}, i_{2}, \ldots, i_{m}\right)$ or alternative by just three special indices $(\lambda, \alpha, j)$,

$$
T_{i_{1} i_{2} \cdots i_{m}} \leftrightarrow T_{\lambda \alpha j}
$$

Rather than trying to write explicitly the components of $T_{\lambda \alpha j}$ as a function of those of $T_{i_{1} i_{2} \cdots i_{m}}$, it is more illuminating to describe the nature of the three new labels. The first one $(\lambda)$ can be any partition of $m$, the second one $(\alpha)$ takes values from 1 to $\mathcal{S}(\lambda, n)$, and the last one $(j)$ goes from 1 to $d(\lambda)$. The reader will correctly infer from here that the following identity holds:

$$
\sum_{\lambda \vdash m} d(\lambda) \mathcal{S}(\lambda, n)=n^{m} .
$$

For a fixed $\alpha, T_{\lambda \alpha j}$ transforms as the irreducible representation $\lambda$ of $S_{m}$ when the $i_{x}$ indices are permuted,

$$
\begin{gathered}
T_{i_{1} i_{2} \cdots i_{m}} \stackrel{\pi}{\rightarrow} T_{\pi\left(i_{1} i_{2} \cdots i_{m}\right)}, \\
T_{\lambda \alpha j} \stackrel{\pi}{\longrightarrow}\left[P_{\lambda}(\pi)\right]_{j j^{\prime}} T_{\lambda \alpha j^{\prime}},
\end{gathered}
$$

where $P_{\lambda}(\pi)$ stands for the matrices of the irreducible representation $\lambda$ of $S_{m}$. This means that the tensor $T$ contains $\mathcal{S}(\lambda, n)$ irreducible representations $\lambda$ of $S_{m}$ (one for each value of $\alpha$ ).

All the above is true for any tensor. It is interesting now to consider the particular scenario where there is not just one tensor but rather a list of tensors $T_{i_{1}, \ldots, i_{m}}^{(1)}, T_{i_{1}, \ldots, i_{m}}^{(2)}, \ldots$, $T_{i_{1}, \ldots, i_{m}}^{(d(\mu))}$ such that

$$
T_{\pi\left(i_{1}, \ldots, i_{m}\right)}^{(a)}=\left[P_{\mu}\left(\pi^{-1}\right)\right]_{a b} T_{i_{1}, \ldots, i_{m}}^{(b)}
$$

for some partition $\mu$ of $m$; the $\phi^{4}$ interactions we considered earlier are the motivation to study this kind of lists of tensors [see text just below Eq. (20), where the $T$ 's were called $c$ 's]. Note that the $P$ matrices can always be made unitary and real, so let us assume for simplicity that they do have these properties. With the $(\lambda, \alpha, j)$ indices it is easy to see what is the most general form of the above list of tensors; it must obey the constraint ${ }^{9}$

$$
\left[P_{\lambda}(\pi)\right]_{j j^{\prime}} T_{\lambda \alpha j^{\prime}}^{(a)}=\left[P_{\mu}(\pi)\right]_{b a} T_{\lambda \alpha j}^{(b)},
$$

and so, according to Shur's lemma from group theory, the components of $T_{\lambda \alpha j}^{(a)}$ are nonzero only if $\lambda=\mu$ and $j=a$, plus their value is independent of $j$,

$$
T_{\lambda \alpha j}^{(a)}=T(\alpha) \delta_{\lambda \mu} \delta_{a j}
$$

The $T(\alpha)$ are free numbers; they are the free parameters in the most general list of tensors $T^{(a)}$ subjected to the constraint in Eq. (38), and there are $\mathcal{S}(\mu, n)$ of them since the index $\alpha$ can go from 1 to this number.

There is one final aspect of lists of tensors with permutation symmetries which is worth looking at, as it will be relevant for the counting of the minimum number of terms in an effective field theory. Consider again a list of tensors which obeys Eq. (38) for some partition $\mu$ of the integer $m(\mu \vdash m)$. This relation states that if we have all the $T_{i_{1}, \ldots, i_{m}}^{(a)}$, we can reproduce the effect of swapping the indices $i_{x}$ by just making linear combinations of the $T_{i_{1}, \ldots, i_{m}}^{(a)}$ with the indices $i_{x}$ unchanged. What is noteworthy is that the opposite is also true: the values of $T_{i_{1}, \ldots, i_{m}}^{(a)}$ can be written down as a linear combination of $T_{\pi\left(i_{1}, \ldots, i_{m}\right)}^{(1)}$ for different $\pi$ (the same is true if we used $T^{(2)}$ or any other component of the list of tensors). This is a consequence of the orthogonality relations among the entries of the unitary (and real) matrices $P_{\mu}(\pi)$ of an irreducible representation:

$$
T_{i_{1}, \ldots, i_{m}}^{(a)}=\frac{d(\mu)}{m !} \sum_{\pi \in S_{m}} P_{\mu}(\pi)_{1 a} T_{\pi\left(i_{1}, \ldots, i_{m}\right)}^{(1)} .
$$

The precise form of this last expression is not particularly relevant; rather, it suffices to keep in mind that all the entries of any of the tensors $T^{(a)}$ can be obtained from the entries of a single one of them ( $T^{(1)}$ for example), given that there is the relation (38). However, we have started with a list of tensors $T^{(a)}$ which, under permutations, transforms as an irreducible representation $\mu$ of $S_{m}$. It is helpful to

\footnotetext{
${ }^{9}$ Since $P_{\mu}\left(\pi^{-1}\right)$ is unitary and real, $\left[P_{\mu}\left(\pi^{-1}\right)\right]_{a b}=\left[P_{\mu}(\pi)\right]_{b a}$.
} 
consider what happens if instead of $\mu$ we had a reducible representation which is a direct sum $\left(\mu_{1}\right)^{r_{1}}+\left(\mu_{2}\right)^{r_{2}}+\cdots$ of distinct irreducible parts $\mu_{i}$ repeated $r_{i}$ times. It turns out that if each $\mu_{i}$ appears at most once in this sum $\left(r_{i}=0\right.$ or $1)$, then it is possible to pick a single combination of the $T^{(a)}$ and generate all $T^{(a)}$ from it as we did before with $T^{(1)}$. On the other hand, if there are $\mu_{i}$ which appear more than once, $\max \left(r_{i}\right)$ linear combinations of the $T^{(a)}$ are needed in order to generate all of them via permutations of the $i_{1}, \ldots, i_{m}$ indices. $^{10}$

In summary, two important facts stand out from this discussion about tensors:

(1) A list of tensors $T^{(a)}$ which transforms under some irreducible representation $\mu$ when its indices are permuted [see Eq. (38)] has $\mathcal{S}(\mu, n)$ independent entries, where $n$ is the number of values each of the tensor indices can take. For multiple irreducible representations $\mu_{i}$, the number of independent entries is given simply by the sum of the numbers $\mathcal{S}\left(\mu_{i}, n\right)$.

(2) A list of tensors $T^{(a)}$ which transforms under a direct sum of irreducible representations $\mu_{i}$, where each $\mu_{i}$ is repeated $r_{i}$ times- $\left(\mu_{1}\right)^{r_{1}}+\left(\mu_{2}\right)^{r_{2}}+\cdots$ - can be reconstructed from just a few of the $T^{(a)}$. Specifically, only $\max \left(r_{i}\right)$ linear combinations of the $T^{(a)}$ are needed for that.

These two observations can be used to count the number of operators and the minimum number of terms in an effective field theory.

\section{Application to operators with repeated fields}

We are now in a position to go back to the discussion of operators with a repeated field $\chi$ which has two indices: a group index $g$ and a flavor index $f$ going from 1 to some number $n$. The product of $m \chi$ 's is either completely symmetric (if $\chi$ is a boson) or completely antisymmetric (if $\chi$ is a fermion),

$$
\chi_{g_{1}}^{f_{1}} \chi_{g_{2}}^{f_{2}} \cdots \chi_{g_{m}}^{f_{m}} \equiv \chi_{\{g\}}^{\{f\}}=( \pm 1)^{\pi} \chi_{\pi\{g\}}^{\pi\{f\}} .
$$

In the Lagrangian, the $\{g\}$ indices contract with some numeric tensors $c_{\{g\}}^{(\alpha)}$ determined from group theory, while the $\{f\}$ indices contract with a parameter tensor $\kappa_{\{f\}}^{(\alpha)}$ (such as the Yukawa matrices in the Standard Model). The extra $\alpha$ index is needed because there might be more than one $c_{\{g\}}$ contraction allowed by the model's symmetries. We then have the expression

\footnotetext{
${ }^{10}$ These linear combinations of the $T^{(a)}$ must be carefully picked; otherwise more than $\max \left(r_{i}\right)$ might be needed in order to generate the full set of tensors $T^{(a)}$.
}

$$
\mathcal{L}=\cdots+\kappa_{\{f\}}^{(\alpha)} c_{\{g\}}^{(\alpha)} \chi_{\{g\}}^{\{f\}}+\cdots
$$

The numerical tensors $c_{\{g\}}^{(\alpha)}$ always obey a symmetry relation of the type

$$
c_{\pi\{g\}}^{(\alpha)}=\left[P\left(\pi^{-1}\right)\right]_{\alpha \beta} c_{\{g\}}^{(\beta)},
$$

where the $P$ matrices form a representation (perhaps reducible) of the permutation group $S_{m}$, and they can be made real and unitary. As a consequence we see that the parameter tensor $\kappa_{\{f\}}^{(\alpha)}$ possesses the following symmetry:

$$
\kappa_{\pi\{f\}}^{(\alpha)}=( \pm 1)^{\pi}\left[P\left(\pi^{-1}\right)\right]_{\alpha \beta} \kappa_{\{f\}}^{(\beta)} .
$$

The product $( \pm 1)^{\pi} P$ is itself a representation $\hat{P}$ of $S_{m}$, so we can write

$$
\kappa_{\pi\{f\}}^{(\alpha)}=\left[\hat{P}\left(\pi^{-1}\right)\right]_{\alpha \beta} \kappa_{\{f\}}^{(\beta)} .
$$

This representation $\hat{P}$ can be decomposed into irreducible representations $\mu \vdash m$ of $S_{m}: \hat{P}=\sum_{\mu \vdash m} r_{\mu} \mu$, where $r_{\mu}$ is the multiplicity of $\mu$ in $\hat{P}^{11}$ The main point of the earlier discussion on tensors is that $\kappa_{\{f\}}^{(\alpha)}$ contains

$$
\sum_{\mu \vdash m} r_{\mu} \mathcal{S}(\mu, n)
$$

free parameters, so this is the total number of operators, and furthermore they all can be written with

$$
\max \left(r_{\mu}\right)
$$

terms. ${ }^{12}$ These comments apply to operators $\chi^{m}$ with a single type of field $\chi$; however, the generalization is trivial for cases where there are more fields, $\chi^{m} \chi^{\prime m^{\prime}} \chi^{\prime \prime m^{\prime \prime}} \ldots$ (namely the above considerations apply separately to each group of repeated fields).

The fact that generally there is more than one group index $g$ is not a problem either. For example, the Standard Model fields carry an index from each of the four groups $S U(3)_{C}, S U(2)_{L}, S U(2)_{l}$, and $S U(2)_{r}$. These indices contract with some symmetry $P^{C}, P^{L}, P^{l}$, and $P^{r}$ so, taken together as a single index $g$, the relevant matrix $P$ is given

\footnotetext{
${ }^{11}$ As mentioned earlier, the notation $\mu \vdash m$ means that $\mu=$ $\left\{\mu_{1}, \mu_{2}, \ldots\right\}$ is a partition of the integer $m$ (so the sum of the $\mu_{i}$ adds up to $m$ ). Therefore $\sum_{\mu \vdash m}$ represents a sum over all partitions $\mu$ of $m$.

${ }^{12}$ Those $\mu$ 's having a Young diagram with more rows than the available flavors $(n)$ should not be considered in this last expression; they are too antisymmetric and as a consequence they are not associated with any operator $[\mathcal{S}(\mu, n)=0]$.
} 
TABLE II. Permutation symmetry of the quark fields $Q$ in $Q Q Q L$-type operators (since there is just one $L$, there is nothing noteworthy about it). The four possible contractions of these fields transform under $S_{3}$ permutations of the $Q$ 's as the sum of irreducible representations $\{3\}+\{2,1\}+\{1,1,1\}(\{2,1\}$ is a two-dimensional representation of $S_{3}$, so in total we do have four contractions). As explained in the text, from this information we can readily conclude that there are $57 Q Q Q L$ operators, which can all be written as a single term in the Lagrangian.

\begin{tabular}{lcc}
\hline \hline & $Q Q Q$ & $L$ \\
\hline$S U(3)_{C}$ & $\square$ & $\square$ \\
$S U(2)_{L}$ & $\square$ & $\square$ \\
$S U(2)_{l}$ & $\square$ & $\square$ \\
$S U(2)_{r}$ & $\square$ & $\square$ \\
Grassmann & $\square$ & $\square$ \\
Total symmetry & $\square^{2} \times \square^{2} \times \square \square=\square \square+\square+\square$ & $\square$ \\
\hline \hline
\end{tabular}

by the Kronecker product $P^{C} \otimes P^{L} \otimes P^{l} \otimes P^{r}$, and $\hat{P}=$ $( \pm 1)^{\pi} P^{C} \otimes P^{L} \otimes P^{l} \otimes P^{r}$.

Take the operators of type $Q Q Q L$ : there are $n_{L} n_{Q}\left(2 n_{Q}^{2}+1\right) / 3$ of them for $n_{Q, L}$ flavors of the fields $Q, L$, and they can all be written with one term. These two conclusions can be obtained by tracking the permutation symmetry of the contraction of the $Q$ 's, as shown in Table II ( $L$ is not repeated, so it is pointless to track its permutation symmetry, which is trivial). In particular, the color indices of the $Q$ 's contract antisymmetrically $(\{1,1,1\})$, while the $S U(2)_{L}$ indices are contracted with the mixed symmetry $\{2,1\}$ as we shall see in Sec. V. ${ }^{13}$ The same is true for the $S U(2)_{l}$ part of the Lorentz group. Since the $Q$ 's are lefthanded fields, we could either ignore entirely the group $S U(2)_{r}$ or alternatively notice that three singlets of $S U(2)_{r}$ contract trivially in a symmetric fashion $(\{3\})$. Finally, we should take into account that these are fermionic fields, so they anticommute $(\{1,1,1\})$. The full symmetry under permutations of the $Q Q Q$ contractions is given by the product of these $S_{3}$ representations $\left(\{3\} \times\{2,1\}^{2} \times\{1,1,1\}^{2}\right)$, which decomposes into the irreducible components $\{3\}+$ $\{2,1\}+\{1,1,1\}$. Note that there are four different ways to contract $Q Q Q L$ [due to the existence of two different $S U(2)_{L}$ contractions as well as two different contractions of the Lorentz indices], and indeed we see that $d(\{3\})+$ $d(\{2,1\})+d(\{1,1,1\})=1+2+1=4$. However, we do not need to write down four terms in the Lagrangian: since each irreducible representation of $S_{3}$ appears only once, we infer that a single term is enough. Furthermore, the total number of operators of the type $Q Q Q L$ is given by the number

$$
\left[\mathcal{S}\left(\square \square, n_{Q}\right)+\mathcal{S}\left(\square, n_{Q}\right)+\mathcal{S}\left(\boxminus, n_{Q}\right)\right] \mathcal{S}\left(\square, n_{L}\right)=\frac{n_{L} n_{Q}\left(2 n_{Q}^{2}+1\right)}{3}
$$

For $n_{Q}=n_{L}=3$, this adds up to 57 complex operators [8]. Historically, the dimension six baryon number violating operators were written as six terms in [1,2], although it is possible to do so with only four [3]. In the particular case of $Q Q Q L$ type operators, they were written as two terms in [1] and in $[7]^{14}$ but, as stated above, these operators require only one term. Notice that Fierz identities were not used to reach this conclusion; spinors and any other field with Lorentz indices are viewed as representations of an $S U(2)_{l} \times S U(2)_{r}$

\footnotetext{
${ }^{13} L$ is an $S U(2){ }_{L}$ doublet, so $Q Q Q$ must transform as a doublet as well. Furthermore, the product of three $S U(2)_{L}$ doublets (one for each $Q$ ) decomposes as $\mathbf{2}+\mathbf{2}+\mathbf{4}$, and the pair of $\mathbf{2}$ 's in this sum transforms as the two-dimensional representation $\{2,1\}$ under $S_{3}$ permutations. This particular case is discussed at the beginning of Sec. V.

${ }^{14}$ In the third arXiv version of this paper, only one term is used.
}

group, and in turn this group is treated in exactly the same way as the gauge group.

Consider another example: the interaction of four right-handed neutrinos mentioned in the introduction $\left(N^{c} N^{c} N^{c} N^{c}\right)$. Each neutrino transforms as a doublet of the $S U(2)_{l}$ group (which is part of the Lorentz group), and we have seen that the two invariant contractions of four doublets have a permutation symmetry $\{2,2\}$. Furthermore, the components of the $N^{c}$ 's are anticommuting fields, so there is a total antisymmetry $\{1,1,1,1\}$ to be taken into consideration. Overall, under permutations, the $N^{c} N^{c} N^{c} N^{c}$ interactions have a symmetry

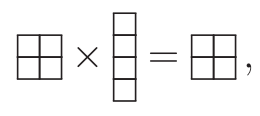

meaning that for $n$ flavors there are 


$$
\mathcal{S}(\boxminus, n)=\frac{n^{2}\left(n^{2}-1\right)}{12}
$$

complex operators of the form $N^{c} N^{c} N^{c} N^{c}$, which can all be written down as a single Lagrangian term. This operator counting matches the one obtained in [36] by other means. More generally, using the same arguments, one concludes that the interactions of $2 m$ right-handed neutrinos have a permutation symmetry

$$
\underbrace{\{2,2, \ldots, 2\}}_{m},
$$

and so there are

$$
\mathcal{S}(\underbrace{\{2,2, \ldots, 2\}}_{m}, n)= \begin{cases}\frac{n !(n+1) !}{m !(m+1) !(n-m+1) !(n-m) !} & n \geq m \\ 0 & n<m\end{cases}
$$

complex operators of the type $\left(N^{c}\right)^{2 m}$, which again can all be written down as a single term in the Lagrangian (for a fixed value of $m$ ).

\section{OPERATORS WITH DERIVATIVES}

\section{A. Handling operator redundancies}

The values of a function and the value of its derivatives do not need to be correlated in any way, so a field $\psi$ and its spacetime derivatives $\partial^{n} \psi$ can be treated as different fields altogether. There is, however, one problem: some operators obtained in this way might be redundant (let us call them $\left.\mathcal{O}_{i}^{0}\right)$, and two Lagrangians differing by such operators will be equivalent.

Faced with this situation, one should work with classes of equivalent Lagrangians $\mathcal{C}_{\mathcal{L}}=\left\{\mathcal{L}+\alpha_{i} \mathcal{O}_{i}^{0}\right\}$ rather than individual Lagrangians. But these $\mathcal{C}_{\mathcal{L}}$ are rather abstract constructions, so it might be better to look at redundancies in effective fields theories as a linear algebra problem. For a given model, the Lagrangian can be viewed as a vector in a vector space spanned by some $z$ operators $\mathcal{O}_{1}, \mathcal{O}_{2}, \ldots$, $\mathcal{O}_{z}$, yet there might exist $r$ combinations $\mathcal{O}_{i}^{0}=\sum_{j} \mathfrak{r}_{i j} \mathcal{O}_{j}$ which are null for practical effects. Hence, there are only $z-r$ meaningful operators $\mathcal{O}_{i}^{\emptyset}=\sum_{j} \mathfrak{m}_{i j} \mathcal{O}_{j}$ which can be selected in more than one way, as long as the block matrix

$$
\left(\begin{array}{c}
\mathfrak{r} \\
\mathfrak{m}
\end{array}\right)
$$

has full rank.

Given $\mathfrak{r}$, the choice of matrix $\mathfrak{m}$ is not unique, but there is a particularly simple possibility: we start with $\mathfrak{m}_{i j}=\delta_{i j}$ and for each row $R$ of $\mathfrak{r}$ (let us call it $\mathfrak{r}_{R}$ ) a line $M$ of $\mathfrak{m}$ $\left(\mathfrak{m}_{M}\right)$ satisfying the condition $\mathfrak{r}_{R} \cdot \mathfrak{m}_{M} \neq 0$ is removed.
In practice, this corresponds to looking at the operators $\mathcal{O}_{j}$ appearing in the redundancy $\sum_{j} \mathfrak{r}_{R j} \mathcal{O}_{j}$ and dropping one of them from any further analysis (as if we were solving the equation $\sum_{j} \mathfrak{r}_{R j} \mathcal{O}_{j}=0$ for one of the $\mathcal{O}_{j}$ 's).

This way of selecting nonredundant operators will always work when there is a single redundancy; however, repeating this simple procedure for multiple redundancies might be problematic, so these more complicated scenarios require caution. ${ }^{15}$ Reference [37] is particularly relevant for this linear algebra view on operator redundancies.

Two types of potentially redundant operators are often considered: (1) those which are zero when the classical equations of motion (EOM) of the fields are applied, and (2) those which are a divergence of a vector field $\left(\partial_{\mu} \mathcal{O}^{\mu}\right)$. Both of these kinds of operators can be ignored only under some assumptions, whose merits will not be assessed in this work. Rather, I will just mention what needs to be done if one wants to factor out these operators. The solution to these problems was already given in the papers $[16,18]$; the following two subsections elaborate on the proposal in [16] to remove EOM redundancies, and they also contain a discussion of a simple adaptation to the procedure mentioned in [18] for factoring out operators of the type $\partial_{\mu} \mathcal{O}^{\mu}$.

It should be stressed at this point that derivatives in a gauge theory always appear through the combination $\partial_{\mu}+i g T^{a} A_{\mu}^{a} \equiv D_{\mu}$, where $D_{\mu}$ is the well-known covariant derivative. For this reason, all derivatives should be seen as being covariant. However, because $\left[D_{\mu}, D_{\nu}\right]$ can be written down with field strength tensors $\mathcal{F}_{\mu \nu}$, in order not to overcount operators one should only consider the completely symmetric part of $D_{\mu_{1}} D_{\mu_{2}} \cdots$ applied to some field $\psi$, in which case the $D_{\mu}$ 's can be seen as commuting with each other-just as normal partial derivatives. To highlight that the term $i g T^{a} A_{\mu}^{a}$ is not important, in this work the symbol $\partial_{\mu}$ will be used instead of $D_{\mu}$.

\section{B. Equations of motion}

It can be shown that the classical equations of motion of a field $\chi$ can be used to reduce the number of nonrenormalizable operators in an effective field theory [38,39]. That is because nonrenormalizable operators proportional to the quantity

$$
\frac{\delta \mathcal{L}_{4}}{\delta \chi}-\partial_{\mu} \frac{\delta \mathcal{L}_{4}}{\delta\left(\partial_{\mu} \chi\right)}
$$

\footnotetext{
${ }^{15}$ As an example, consider three operators $\mathcal{O}_{1,2,3}$. If there is a redundancy $\mathcal{O}_{1}+\mathcal{O}_{2}+\mathcal{O}_{3}$, we might deal with it by dropping the operator $\mathcal{O}_{3}$, in which case we would consider that the space of all nonequivalent Lagrangians is generated by $\mathcal{O}_{1}$ and $\mathcal{O}_{2}$. If there is a second redundancy $-\mathcal{O}_{1}+\mathcal{O}_{2}+\mathcal{O}_{3}$, one cannot drop $\mathcal{O}_{3}$ (since we have done so already), but more importantly we cannot simply drop $\mathcal{O}_{2}$ either, and say that $\mathcal{O}_{1}$ alone generates all nonequivalent Lagrangians. That is because the vectors $\mathcal{O}_{1}+$ $\mathcal{O}_{2}+\mathcal{O}_{3},-\mathcal{O}_{1}+\mathcal{O}_{2}+\mathcal{O}_{3}$, and $\mathcal{O}_{1}$ are not linearly independent.
} 
or its derivatives, where $\mathcal{L}_{4}$ is the renormalizable part of a Lagrangian, do not affect the $S$-matrix. The standard approach to these redundancies is to remove the operators with the highest number of derivatives [the second term in expression (55)], since these can be traded by other operators with fewer derivatives [the first term in expression (55)].

The authors of [16] pointed out that these redundancies due to the equations of motion are best seen if we decompose the field derivatives $\partial^{i} \chi$ into irreducible representations of the Lorentz group. ${ }^{16}$ For example, the 16 second derivatives of a scalar $\phi$ transform under $S U(2)_{l} \times S U(2)_{r}$ as

$$
\partial^{2} \phi=(0,0)+(0,1)+(1,0)+(1,1),
$$

but apart from $(1,1)$ (with nine components), these irreducible representations of the Lorentz are redundant. The reason is the following. The parts $(0,1)$ and $(1,0)$ are antisymmetric under permutation of the two derivatives, so they can be written with the field stress tensors, $\left[\partial^{2} \phi\right]_{(0,1)+(1,0)} \propto \mathcal{F} \phi$, so we should discard them. On the other hand, the equation of motion of $\phi$ equates $\left[\partial^{2} \phi\right]_{(0,0)}=$ $\square \phi$ to terms without derivatives so, if we follow the rule of keeping terms with as few derivatives as possible, only the $(1,1)$ part of $\partial^{2} \phi$ needs to be kept.

All four first derivatives of $\phi$ which transform as

$$
\partial \phi=\left(\frac{1}{2}, \frac{1}{2}\right)
$$

are to be kept as well, but in the case of a left-handed fermion $\psi$ the situation is different. The eight components of $\partial \psi$ transform as

$$
\partial \psi=\left(1, \frac{1}{2}\right)+\left(0, \frac{1}{2}\right)
$$

yet the equations of motion of $\psi$ relate $[\partial \psi]_{\left(0, \frac{1}{2}\right)}$ to a quantity with no derivatives, so we should keep only $[\partial \psi]_{\left(1, \frac{1}{2}\right)}$. Adding one more derivative, in analogy to the scalar case, we should worry only about $\left[\partial^{2} \psi\right]_{\left(\frac{3}{2}, 1\right)}$.

This procedure works well not just for Hilbert series calculations [16] but also for the more straightforward approach to operator counting being described in this work. In practice, the EOM degeneracies are taken into account by introducing a tower of new fields representing the nonredundant parts of $\partial \chi, \partial^{2} \chi, \ldots, \partial^{i} \chi$, for every standard model field $\chi$. They have the same gauge quantum numbers

\footnotetext{
${ }^{16}$ The exponent $i$ in a derivative refers to the product of $\partial \times$ $\partial \times \cdots$ with $i$ factors. Similarly, $\partial^{2}=\partial \times \partial$. The exponent only refers to a spacetime index if a Greek letter is used ( $\partial^{\mu}$ for instance).
}

as $\chi$ but different Lorentz representations. Since we are expanding significantly the number of fields in the effective field theory, this has the adverse effect of increasing the computational complexity of the calculations. Nevertheless, the procedure to handle the equations of motion is conceptually very simple.

In any case, one must know what are the components of the field derivatives to be kept. For a generic field $\chi$ we just saw that there are two considerations to have in mind:

(i) We want only the $\partial^{i} \chi$ components which cannot be written with field strength tensors; hence they correspond to those components which are completely symmetric under permutations of the derivatives. We may represent them by the expression $\left\{\partial^{i}\right\} \chi$ which, in general, transforms as a reducible representation of the Lorentz group-for example, $\left\{\partial^{4}\right\} \phi=(2,2)+(1,1)+(0,0)$.

(ii) Some components of $\left\{\partial^{i}\right\} \chi$ are also redundant for another reason. If we represent the equation of motion of $\chi$ with the notation

$$
\begin{gathered}
\left\langle\partial^{x} \chi\right\rangle=\cdots(x=1 \text { for fermions and } x=2 \\
\text { for bosons }),
\end{gathered}
$$

the operator $\left\langle\partial^{x} \chi\right\rangle$ and its derivatives should also be discarded.

However, there is a difficulty with this two-stage reduction of operators: we cannot just take the irreducible Lorentz representations in $\left\{\partial^{i}\right\} \chi$ and remove all those in $\partial^{i-x}\left\langle\partial^{x} \chi\right\rangle$, because some of the latter components are antisymmetric under permutations of the derivatives, and therefore can be written with the field strength tensor $\mathcal{F}_{\mu \nu}$. So in reality the terms to be retained are

$$
\begin{aligned}
& {\left[\partial^{i} \chi\right]_{\text {keep }}=\left\{\partial^{i}\right\} \chi \text { minus the components in }} \\
& {\left[\partial^{i-x}\left\langle\partial^{x} \chi\right\rangle\right]_{\text {all } \partial \text { 's sym. contracted }} \text {. }}
\end{aligned}
$$

Figuring out what are the components of $\partial^{i} \chi$ to keep is not a trivial exercise; it turns out that for a scalar $\phi=(0,0)$, a left-hand fermion $\psi=\left(\frac{1}{2}, 0\right)$ and a field strength tensor $F=\{1,0\}$ (recall that $\mathcal{F}=F+F^{*}$ ) only the highest spin component should be retained, i.e.,

$$
\begin{gathered}
{\left[\partial^{i} \phi\right]_{\text {keep }}=\left(\frac{i}{2}, \frac{i}{2}\right),} \\
{\left[\partial^{i} \psi\right]_{\text {keep }}=\left(\frac{i+1}{2}, \frac{i}{2}\right),} \\
{\left[\partial^{i} F\right]_{\text {keep }}=\left(\frac{i+2}{2}, \frac{i}{2}\right) .}
\end{gathered}
$$

The rest of this subsection discusses this result (see also [40]).

Let us consider first a scalar $\phi$. It is not hard to show that 


$$
\begin{aligned}
\left\{\partial^{i}\right\} \phi= & \left(\frac{i}{2}, \frac{i}{2}\right)+\left(\frac{i}{2}-1, \frac{i}{2}-1\right)+\left(\frac{i}{2}-2, \frac{i}{2}-2\right) \\
& +\cdots
\end{aligned}
$$

On the other hand, in order to calculate the Lorentz transformation properties of the components $\partial^{i-2}\left\langle\partial^{2} \phi\right\rangle$ which need to be removed, we may want to apply the $i-2$ derivatives in a completely symmetric way:

$$
\left\{\partial^{i-2}\right\}\left\langle\partial^{2} \phi\right\rangle=\left(\frac{i}{2}-1, \frac{i}{2}-1\right)+\left(\frac{i}{2}-2, \frac{i}{2}-2\right)+\cdots .
$$

It is then tempting to subtract these Lorentz irreducible representations from the ones in Eq. (64) and conclude that the only nonredundant piece of $\left\{\partial^{i}\right\} \phi$ is the one transforming as $\left(\frac{i}{2}, \frac{i}{2}\right)$. The last statement is true, but one should keep in mind that this argument only works because none of the components of $\left\{\partial^{i-2}\right\}\left\langle\partial^{2} \phi\right\rangle$ is proportional to $\mathcal{F}_{\mu \nu}$; in other words, all components of $\left\{\partial^{i-2}\right\}\left\langle\partial^{2} \phi\right\rangle$ have a part which is completely symmetric under all permutations of all $i$ derivatives. ${ }^{17}$

For derivatives of a left-handed fermion, the transformation properties under Lorentz transformations are as follows:

$$
\begin{aligned}
\left\{\partial^{i}\right\} \psi= & \left(\frac{i+1}{2}, \frac{i}{2}\right)+\left(\frac{i+1}{2}-1, \frac{i}{2}-1\right)+\left(\frac{i+1}{2}-2, \frac{i}{2}-2\right)+\cdots \\
& +\left(\frac{i-1}{2}, \frac{i}{2}\right)+\left(\frac{i-1}{2}-1, \frac{i}{2}-1\right)+\left(\frac{i-1}{2}-2, \frac{i}{2}-2\right)+\cdots, \\
\left\{\partial^{i-1}\right\}\langle\partial \psi\rangle= & \left(\frac{i+1}{2}-1, \frac{i}{2}-1\right)+\left(\frac{i+1}{2}-2, \frac{i}{2}-2\right)+\cdots \\
& +\left(\frac{i-1}{2}, \frac{i}{2}\right)+\left(\frac{i-1}{2}-1, \frac{i}{2}-1\right)+\left(\frac{i-1}{2}-2, \frac{i}{2}-2\right)+\cdots
\end{aligned}
$$

Assuming again that all components of $\left\{\partial^{i-1}\right\}\langle\partial \psi\rangle$ are, at least in part, fully symmetric, the only nonredundant component of $\partial^{i} \psi$ is $\left(\frac{i+1}{2}, \frac{i}{2}\right)$, with maximum spin. This is the only part of the right-hand side of Eq. (66) which remains after subtracting the right-hand side of Eq. (67).

Finally, we have to consider what happens to derivatives of field strength tensors $\mathcal{F}$. As before, one might try to compute the irreducible Lorentz representations associated with the components $\left\{\partial^{i}\right\} \mathcal{F}$ (let us call these components $A L L)$ and remove those which appear in the equations of motion $\left\{\partial^{i-1}\right\}\langle\partial \mathcal{F}\rangle$ (we may call this set of components $E O M)$. The trouble is that in some cases the $i+1$ derivatives in $\left\{\partial^{i}\right\} \mathcal{F}$ and $\left\{\partial^{i-1}\right\}\langle\partial \mathcal{F}\rangle^{18}$ are not contracted in a fully symmetric way (they form a set $A S$ ). Given that all derivatives are in reality covariant, these $\left\{\partial^{i}\right\} \mathcal{F}$ components can be written with two or more field strength tensors

\footnotetext{
${ }^{17}$ We know that the two derivatives in $\left\langle\partial^{2} \phi\right\rangle=\eta^{\mu \nu} \partial_{\mu} \partial_{\nu}$ are contracted symmetrically, and so are the remaining ones in $\left\{\partial^{i-2}\right\}$ (obviously). Therefore the $(i+1) ! /[3 !(i-2) !]$ components of $\left\{\partial^{i-2}\right\}\left\langle\partial^{2} \phi\right\rangle$ transform as a representation $\{i-2\} \times\{2\}$ of the group $S_{i-2} \times S_{2}$ under permutations of the derivatives. From this information alone, one cannot conclude that under permutations of the bigger group $S_{i} \supset S_{i-2} \times S_{2}$ all these components transform as a fully symmetric representation $\{i\}$ (it is conceivable that they could also transform as $\{i-1,1\}$ or $\{i-2,2\})$.

${ }^{18}$ Note that $\mathcal{F}$ already contains one derivative.
}

$\mathcal{F}$, so they are redundant. We wish to calculate the elements of $A L L$ which are neither in EOM nor in $A S$, but this is complicated by the fact that these last two sets intersect (see Fig. 1). The number of elements of each set, as a function of $i$, is the following:

$$
\begin{array}{r}
6 \mathcal{S}(\{i\}, 4)=(i+3)(i+2)(i+1) \quad(\# A L L), \\
\mathcal{S}(\{i, 1,1\}, 4)=\frac{(i+3)(i+1) i}{2} \quad(\# A S), \\
4 \mathcal{S}(\{i-1\}, 4)=\frac{2(i+2)(i+1) i}{3} \quad(\# E O M) .
\end{array}
$$

The sets $E O M$ and $A S$ cannot be disjoint simply from the fact that \#AS+\#EOM can be larger than \#ALL. Up to large values of $i$, it is possible to check explicitly that the intersection of $E O M$ and $A S$ contains

$$
\frac{(i+1) i(i-1)}{6} \quad[\#(A S \cap E O M)]
$$

elements, and from here we can conclude that there are

$$
2(i+3)(i+1) \quad\{\#[A L L \backslash(A S \cup E O M)]\}
$$

components of $\left\{\partial^{i}\right\} \mathcal{F}$ which are not redundant. The maximum spin components, transforming under the Lorentz representation $\left(\frac{i+2}{2}, \frac{i}{2}\right)+\left(\frac{i}{2}, \frac{i+2}{2}\right)$, are certainly 


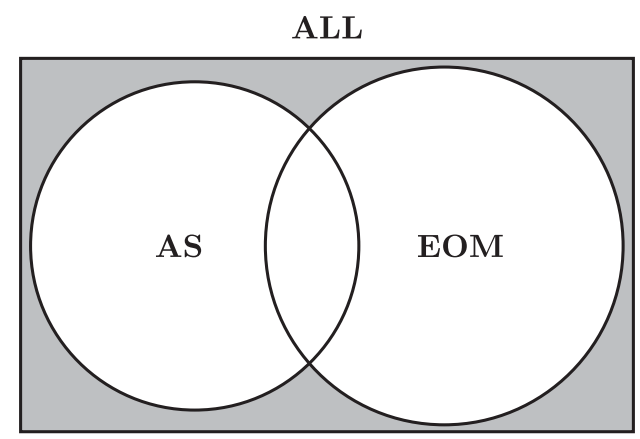

FIG. 1. The set of all $\left\{\partial^{i}\right\} \mathcal{F}$ components $(A L L)$ includes components which are redundant due to antisymmetric contractions of the $i+1$ derivatives (set $A S$ ) and/or due to the equations of motion (set $E O M$ ). In order to remove these redundancies and obtain the truly important $\left\{\partial^{i}\right\} \mathcal{F}$ components (gray part of the diagram), one must understand which elements of $A L L$ are in both $A S$ and EOM. (For $i=6$, the diagram shown here faithfully represents the relative size of the sets and their intersections.)

not contained in either EOM nor $A S$, and they are precisely $2(i+3)(i+1)$ in total. Hence, one can conclude that these are the only components of $\left\{\partial^{i}\right\} \mathcal{F}$ to keep.

\section{Integration by parts}

There is another kind of operator redundancy. The action is calculated by integrating the Lagrangian density over all spacetime, so what matters are not the operators $\mathcal{O}$ themselves but rather their spacetime integral. If $\mathcal{O}$ is a divergence, $\mathcal{O}=\partial^{\mu} \mathcal{O}_{\mu}$, and the value of $\mathcal{O}_{\mu}$ drops to zero fast enough at large distances/times, then by the divergence theorem

$$
\int_{\mathcal{M}} \mathcal{O} d^{4} x=0
$$

where $\mathcal{M}$ is some infinitely large volume of spacetime. Putting aside the possibility that the above integral might not always vanish, operators of the form $\mathcal{O}=\partial^{\mu} \mathcal{O}_{\mu}$ should be discarded. This is often seen as an integration by parts redundancy: if the sum of two operators $\mathcal{O}_{\mu}^{(1)}$ and $\mathcal{O}_{\mu}^{(2)}$ is a total divergence, an integration by parts reveals that $\mathcal{O}_{\mu}^{(1)}$ is equivalent to $-\mathcal{O}_{\mu}^{(2)}$.

We may track the operators which are total derivatives by introducing a dummy field $\mathcal{D}$ in the effective field theory representing total derivatives, so that operators of the form $\mathcal{D}(\cdots)$ should be factored out. However, some of these latter operators are redundant [18], because there are linear combinations of them which add up to zero. Unfortunately, it does not seem easy to detect these relations among operators which are total divergences.

Nevertheless, the authors of [18] managed to count them with the Hilbert series method and conformal field theory; they also offered an interesting interpretation of their calculation which does not rely on these particular theoretical tools. It goes as follows, using the language of differential forms. The redundant operators $\mathcal{O}=\partial^{\mu} \mathcal{O}_{\mu}$ are associated with those differential 4-forms $\omega^{(4), \text { red }}$ which are exact, meaning that they are the differential of some 3-form $\omega^{(3)}: \omega^{(4) \text {,red }}=d \omega^{(3)}$. Nevertheless, one cannot simply consider all $\omega^{(3)}$ because the differential of some 3 -forms is identically zero, i.e., $d \omega^{(3) \text {,red }}=0$. In turn, according to Poincaré's lemma, these "redundancies of the redundancies" are associated with all those 3-forms which are the differentials of a 2 -form $\omega^{(2)}: \omega^{(3), \text { red }}=d \omega^{(2)}$. This recursive argument goes on, but it eventually stops because of the dimensionality of spacetime (four) when we reach 0 -forms, which are pure functions.

Let us rephrase this argument in the language of operators. In general, a differential $i$-form $\omega^{(i)}$ can be written as

$$
\omega^{(i)}=\mathcal{O}_{\mu_{1} \mu_{2} \cdots \mu_{i}} d x^{\mu_{1}} \wedge d x^{\mu_{2}} \ldots \wedge d x^{\mu_{i}},
$$

where $\mathcal{O}_{\mu_{1} \mu_{2} \cdots \mu_{i}}$ is a completely antisymmetric tensor under an exchange of indices; to highlight this fact, from now on square brackets will be applied to its indices. Applying the differential operator $4-i$ times to $\omega^{(i)}$ yields

$$
d^{4-i} \omega^{(i)} \propto \partial^{\mu_{1}} \partial^{\mu_{2}} \cdots \partial^{\mu_{i}} \mathcal{O}_{\left[\mu_{1} \mu_{2} \cdots \mu_{i}\right]} d x^{0} \wedge d x^{1} \wedge d x^{2} \wedge d x^{3}
$$

If $i>1$, the expressions $\partial^{\mu_{1}} \partial^{\mu_{2}} \cdots \partial^{\mu_{i}} \mathcal{O}_{\left[\mu_{1} \mu_{2} \cdots \mu_{i}\right]}$ are identically zero, but even so it is crucial that we keep track of them. Consider the set $\{\mathcal{O}\}$ of all scalar operators allowed by the model's symmetries, as well as the set of all operators $\left\{\mathcal{O}_{\left[\mu_{1} \mu_{2} \cdots \mu_{i}\right]}\right\}$ with $i=1,2,3,4$ free spacetime indices, which are completely antisymmetric under the exchange of these indices. The nonredundant operators are those in the set

$$
\begin{aligned}
& \{\mathcal{O}\} \backslash\left(\{ \partial ^ { \mu } \mathcal { O } _ { \mu } \} \backslash \left(\{ \partial ^ { \mu } \partial ^ { \nu } \mathcal { O } _ { [ \mu \nu ] } \} \backslash \left(\left\{\partial^{\mu} \partial^{\nu} \partial^{\rho} \mathcal{O}_{[\mu \nu \rho]}\right\} \backslash\right.\right.\right. \\
& \left.\left.\left.\quad\left\{\partial^{\mu} \partial^{\nu} \partial^{\rho} \partial^{\sigma} \mathcal{O}_{[\mu \nu \rho \sigma]}\right\}\right)\right)\right) .
\end{aligned}
$$

In summary, all linear combinations of the operators $\{\mathcal{O}\}$ are nonzero, but we wish to remove the degeneracies $\left\{\partial^{\mu} \mathcal{O}_{\mu}\right\}$. Unfortunately, this last set includes some operators which are identically zero, hence the need to include the remaining sets in expression (76), containing only null operators. If all we want is to count the number of nonredundant operators up to some dimension $d$, then the result is ${ }^{19}$

\footnotetext{
${ }^{19}$ In four-dimensional spacetime, there is a one-to-one correspondence between the operators $\mathcal{O}_{\mu}$ and $\mathcal{O}_{[\mu \nu \rho]}$ with the same dimension, as well as between the operators $\mathcal{O}$ and $\mathcal{O}_{[\mu \nu \rho \sigma]}$. Therefore expression (77) is the same as $\left(\# \mathcal{O}^{\operatorname{dim} \leq d}\right)-$ $\left(\# \mathcal{O}_{\mu}^{\operatorname{dim} \leq d-1}\right)+\left(\# \mathcal{O}_{[\mu \nu]}^{\operatorname{dim} \leq d-2}\right)-\left(\# \mathcal{O}_{\mu}^{\operatorname{dim} \leq d-3}\right)+\left(\# \mathcal{O}^{\operatorname{dim} \leq d-4}\right)$.
} 
TABLE III. Relevant types of operators of the generic form $\partial^{4} S^{4}$, where $S$ is a scalar with trivial gauge quantum numbers. As explained in the main text, their total number is obtained by counting the operators with no $\mathcal{D}$ 's and subtracting those with one $\mathcal{D}$. However, this naive operation subtracts too many operators. To compensate for this, one must put back the operators with two $\mathcal{D}$ 's, remove those with three $\mathcal{D}$ 's, and finally put back those with four $\mathcal{D}$ 's.

\begin{tabular}{|c|c|c|}
\hline \#D's & Operator type & Symmetry of the fields $\left(S, \partial S, \partial^{2} S\right)$ \\
\hline \multirow[t]{3}{*}{0} & $S S\left(\partial^{2} S\right)\left(\partial^{2} S\right)$ & $(\square \square,-, \square \square)$ \\
\hline & $S(\partial S)(\partial S)\left(\partial^{2} S\right)$ & $(\square, \square \square, \square)$ \\
\hline & $(\partial S)(\partial S)(\partial S)(\partial S)$ & $(-, \square \square,-)+(-, \boxminus,-)+(-, \Theta,-$ \\
\hline \multirow[t]{2}{*}{1} & $\mathcal{D} S S(\partial S)\left(\partial^{2} S\right)$ & $(\square \square, \square, \square)$ \\
\hline & $\mathcal{D} S(\partial S)(\partial S)(\partial S)$ & $(\square, \square \square,-)+(\square, \square,-)+(\square, \Theta,-)$ \\
\hline 2 & $\mathcal{D D} S S(\partial S)(\partial S)$ & $2(\square, \boxminus,-)$ \\
\hline 3 & $\mathcal{D D D} \operatorname{SSS}(\partial S)$ & $(\square \square, \square,-)$ \\
\hline 4 & $\mathcal{D D D D D S S S S}$ & $\left(\begin{array}{l|l}\square & \square\end{array},-,-\right)$ \\
\hline
\end{tabular}

$$
\begin{aligned}
& \left(\# \mathcal{O}^{\operatorname{dim} \leq d}\right)-\left(\# \mathcal{O}_{\mu}^{\operatorname{dim} \leq d-1}\right)+\left(\# \mathcal{O}_{[\mu \nu]}^{\operatorname{dim} \leq d-2}\right)-\left(\# \mathcal{O}_{[\mu \nu \rho]}^{\operatorname{dim} \leq d-3}\right) \\
& \quad+\left(\# \mathcal{O}_{[\mu \nu \rho \sigma]}^{\operatorname{dim} \leq d-4}\right) .
\end{aligned}
$$

Each of the five numbers in this sum can be calculated by directly counting how many operators there are with the indicated transformation property under the Lorentz group: Under $S U(2)_{l} \times S U(2)_{r}$, an operator with no subscript must transform as $(0,0)$, while those with the subscripts $\mu$, $[\mu \nu],[\mu \nu \rho]$, and $[\mu \nu \rho \sigma]$ transform as $\left(\frac{1}{2}, \frac{1}{2}\right),(1,0)+(0,1)$, $\left(\frac{1}{2}, \frac{1}{2}\right)$, and $(0,0)$, respectively.

Alternatively, one can avoid handling operators which are not scalars by introducing the derivative $\mathcal{D}$ as a standalone field as mentioned earlier, with all the expected properties, except that it anticommutes (making it a Grassmann field). With this dummy field,

$$
\begin{gathered}
\# \mathcal{O}_{\mu}^{\operatorname{dim} \leq d-1}=\#\left(\mathcal{D}^{\mu} \mathcal{O}_{\mu}\right)^{\operatorname{dim} \leq d}, \\
\# \mathcal{O}_{[\mu \nu]}^{\operatorname{dim} \leq d-2}=\#\left(\mathcal{D}^{\mu} \mathcal{D}^{\nu} \mathcal{O}_{[\mu \nu]}\right)^{\operatorname{dim} \leq d}, \\
\# \mathcal{O}_{[\mu \nu \rho]}^{\operatorname{dim} \leq d-3}=\#\left(\mathcal{D}^{\mu} \mathcal{D}^{\nu} \mathcal{D}^{\rho} \mathcal{O}_{[\mu \nu \rho]}\right)^{d i m \leq d}, \\
\# \mathcal{O}_{[\mu \nu \rho \sigma]}^{\operatorname{dim} \leq d-4}=\#\left(\mathcal{D}^{\mu} \mathcal{D}^{\nu} \mathcal{D}^{\rho} \mathcal{D}^{\sigma} \mathcal{O}_{[\mu \nu \rho \sigma]}\right)^{\operatorname{dim} \leq d},
\end{gathered}
$$

where the $\mathcal{O}$ 's are assumed not to contain any $\mathcal{D}$. Due to these identities, it becomes necessary to track only the scalar operators up to dimension $d$, and expression (77) now reads

$$
\sum_{i=0}^{4}(-1)^{i}\left(\# \mathcal{O}^{\operatorname{dim} \leq d} \quad \text { with } \quad i \mathcal{D}^{\prime} s\right) .
$$

Note that this only works if $\mathcal{D}$ is a Grassmann field, as the subscripts $[\mu \nu \cdots]$ of the operators in expression (77) are completely antisymmetrized. In fact, if $\mathcal{D}$ was a commuting field, then $\mathcal{D}^{\mu} \mathcal{D}^{\nu} \cdots \mathcal{O}_{[\mu \nu \cdots]}$ would be identically 0 .

As an example, Table III lists all types of operators with four derivatives and four gauge invariant scalars $S$. From the permutation symmetries of $S, \partial S$, and $\partial^{2} S$, we can compute that there are the following numbers of operators, assuming $n$ flavors of $S$ (see Sec. III):

$\# \mathcal{O}=\frac{1}{12} n^{2}\left(11 n^{2}+12 n+13\right) \quad$ (operators with 0D's),

$\# \mathcal{O}_{\mu}=\frac{1}{6} n^{2}\left(7 n^{2}+3 n+2\right) \quad($ operators with $1 \mathcal{D})$,

$\# \mathcal{O}_{[\mu \nu]}=\frac{1}{2} n^{2}\left(n^{2}-1\right) \quad$ (operators with 2D's),

$\# \mathcal{O}_{[\mu \nu \rho]}=\frac{1}{6} n^{2}(n+1)(n+2) \quad$ (operators with 3D's),

$$
\# \mathcal{O}_{[\mu \nu \rho \sigma]}=\frac{1}{24} n(n+1)(n+2)(n+3)
$$

(operators with 4D's).

It follows that there is a total of $\frac{1}{8} n\left(n^{3}+2 n^{2}+3 n+2\right)$ operators of the generic form $\partial^{4} S^{4}$.

Crucially, in order to arrive at this counting, one must be able to compute the permutation symmetries shown in Table III. This can be done as discussed previously, and to demonstrate it consider, for example, the operators of the type $(\partial S)^{4}$. The field $\partial S$ is gauge invariant, but it transforms under the Lorentz group $S U(2)_{l} \times S U(2)_{r}$ as a bidoublet. We have already seen that the $S U(2)$ invariant contractions of four doublets transform under $S_{4}$ permutations as $\{2,2\}$ (see also the next section), hence this statement holds for both $S U(2)_{l}$ and $S U(2)_{r}$. Taking into 
account these two groups, we conclude that the contractions of the four $\partial S$ 's transform under the representation $\{2,2\} \times\{2,2\}$, and this product decomposes into the sum $\{4\}+\{2,2\}+\{1,1,1,1\}$ of $S_{4}$ irreducible representations, in agreement with Table III.

As a second example, we shall look into those operators of type $\mathcal{D D} S S(\partial S)(\partial S)$. The two $\mathcal{D}$ 's must contract symmetrically because there is just one copy of this dummy field. Each $\mathcal{D}$ is a bidoublet of $S U(2)_{l} \times S U(2)_{r}$, so we are looking at the product $\left(\frac{1}{2}, \frac{1}{2}\right) \times\left(\frac{1}{2}, \frac{1}{2}\right)=(1,1)_{S}+(0,0)_{S}+$ $(1,0)_{A}+(0,1)_{A}$. Given that $\mathcal{D}$ is a Grassmann field, we infer that $\mathcal{D D}$ transforms as $(1,0)+(0,1)$. Therefore $(\partial S)(\partial S)$ must transform as either $(1,0)$ or $(0,1)$ - these are two valid possibilities, and crucially in both case the $\partial S$ 's contract antisymmetrically. The $S$ 's always contract in a symmetric way (after all, $S$ is a Lorentz and gauge invariant field); therefore we deduce that for the two possible contractions of the Lorentz indices, the relevant permutation symmetry of $(S, \partial S)$ is $(\{2\},\{1,1\})$.

\section{DISCUSSION OF THE ALGORITHM AND SOME RESULTS}

\section{A. Implementation in a computer code}

The approach described in this work makes it possible to count operators of an effective field theory, and also to extract some extra information about them. However, in order to implement it, for a representation $R$ of some Lie group $G$ one must be able to decompose the tensor product $R^{m}=R \times R \times \cdots \times R$ into irreducible representations of $G \times S_{m}$. It is not enough to know the decomposition of such products into irreducible representations of the Lie group $G$ alone.

There is a widely known technique involving Young tableaux to extract this information when $G$ is a special unitary group $S U(p)$ and $R$ is its fundamental representation. Any $S U(p)$ representation can be labeled by a partition or a Young diagram, much as the representations of the permutation group, with two caveats:

(1) Columns with $p$ rows can be ignored, so two Young diagrams differing only by such columns stand for the same $S U(p)$ representation;

(2) Diagrams with more than $p$ rows are not associated with any $S U(p)$ representation.

If $R$ is the fundamental representation of $S U(p)$, it turns out that the tensor product $R^{m}=R \times R \times \cdots \times R$ decomposes into the sum of all representations $\lambda_{\lambda}$ of the group $S U(p)_{S_{m}}$ ( $\lambda$ is a partition of $m$ with at most $p$ rows). For example, in the case of four $S U(2)$ doublets, $\lambda$ can be $\{4\},\{3,1\}$, or $\{2,2\}$, so we obtain the decomposition

$$
\mathbf{2} \times \mathbf{2} \times \mathbf{2} \times \mathbf{2}=\mathbf{5}_{\{4\}}+\mathbf{3}_{\{3,1\}}+\mathbf{1}_{\{2,2\}},
$$

because the partitions $\lambda=\left\{\lambda_{1}\right\}$ and $\lambda=\left\{\lambda_{1}, \lambda_{2}\right\}$ correspond to the $\left(\lambda_{1}+1\right)$ - and $\left(\lambda_{1}-\lambda_{2}+1\right)$-dimensional representations of $S U(2)$, respectively. Note that $\{4\}$, $\{3,1\}$, or $\{2,2\}$ are irreducible representations of $S_{4}$ with dimensions 1,3 , and 2 , respectively. So on the right-hand side of Eq. (88) there is a total of $1 \times 5+3 \times 3+2 \times 1=$ $16=2^{4}$ components, as expected. One can alternatively express the product of four doublets as

$$
\mathbf{2} \times \mathbf{2} \times \mathbf{2} \times \mathbf{2}=\mathbf{5}+\mathbf{3}+\mathbf{3}+\mathbf{3}+\mathbf{1}+\mathbf{1},
$$

but in doing so we are erasing critical information from Eq. (88).

When discussing previously the $Q Q Q L$ operator, it was stated that the $S U(2)_{L}$ indices of the $Q$ 's transform as $\{2,1\}$ under $S_{3}$ permutations. I will now mention two proofs of this statement. On the one hand, we just saw that the two singlets in the product of four doublets of $S U(2)$ transform as $\{2,2\}$ under $S_{4}$. However, in $Q Q Q L$ we are dealing with three equal doublets $(Q)$ plus a fourth doublet $(L)$ which is a distinct field. We therefore do not care about all $4 !=24$ permutations of the four doublets but rather we are only interested in the six permutation of the subgroup $S_{3} \times S_{1} \subset S_{4}$. It turns out that the irreducible representation $\{2,2\}$ of $S_{4}$ transforms as $\{2,1\} \times\{1\}$ when restricted to this subgroup, or simply $\{2,1\}$ if we ignore the trivial $S_{1}$ group.

An alternative method of arriving at this conclusion is to repeat the decomposition show in Eq. (88), but this time for three doublets only. With the same arguments as before, we get that

$$
\mathbf{2} \times \mathbf{2} \times \mathbf{2}=\mathbf{4}_{\{3\}}+\mathbf{2}_{\{2,1\}} .
$$

Each of the three doublets here stands for one of the $Q$ 's, and in order for $Q Q Q$ to form an $S U(2)_{L}$ invariant with $L$, which is also a doublet, we must select only the doublet contraction of $Q Q Q$, which transforms as $\{2,1\}$ under $S_{3}$ permutations.

The tight connection between $S U(p)$ and the permutation group which was just mentioned is all that is needed to count operators of some models (including SMEFT). However, it is worth pointing out that this type of decomposition can be calculated for any representation of any group, including discrete ones. The LIE program [41] does this efficiently for any simple Lie group, referring to it as a computation of a plethysm. The algorithm described in LIE's manual was implemented in the SUSYNO package for Mathematica [27]. This latter program also contains several other functions related to Lie algebras and the permutation group $S_{n}{ }^{20}$ The first version of the SYM2INT package [28] uses them to list the operators without derivatives (nor field

\footnotetext{
${ }^{20}$ The group theory part of this code has recently been separated and enlarged in a separate package called GROUPMATH [42]. All the computations involving Lie groups and/or the permutation group $S_{n}$ which are mentioned in this work can be performed with it.
} 
strength tensors) of any effective field theory. This can be done up to some arbitrary mass dimension. Adapting the solutions in the literature $[16,18]$ to tackle the problems inherent to derivatives, the latest version of SYM2INT can list automatically all operators in an effective field theory, including those with derivatives and field strength tensors.

\section{B. Comparison with other methods and computer codes}

All SMEFT operators up to dimension 15 were counter and characterized in the way described here in under two hours on a standard laptop computer (details of these results can be found below, at the end of this section). All the numbers and expressions given in [18] (and in the accompanying data files) were successfully reproduced with the alternative method described here.

Two other noteworthy codes should be mentioned. The first one is DEFT [37]: rather than just calculate the number of operators and their symmetries, this code works with the actual operators, performing the gauge and Lorentz index contractions explicitly. Dealing with operator redundancies associated with derivatives then becomes a linear algebra problem. Having the operators written down explicitly is clearly something very useful for model builders, and it opens up several possibilities, such as of implementing in different ways the operator redundancies discussed previously. One downside of the more ambitious scope of DEFT is that it takes more time to perform these calculations - as a point of reference, the authors of [37] were able to calculate SMEFT up to dimension eight for one fermion generation, which corresponds to roughly a third of the number of SMEFT operators up to dimension six, with three generations.

The second noteworthy code is BASISGEN [43]. It counts operators, and does so with the basic approach of multiplying all fields together, and retaining only the gauge and Lorentz invariant solutions. This is essentially the same method of counting operators as the one described in this paper and implemented in SYM2INT (this was called the traditional method in Sec. II). However, repeated fields are handled differently, and this has significant impact on the computational time for models with multiple flavors. Instead of $n$ flavors of a field $\psi$, BASISGEN considers $n$ distinct fields $\psi, \psi^{\prime}, \psi^{\prime \prime}, \ldots$, with the same quantum numbers, meaning that flavor indices are expanded. From this perspective all fields have a single flavor so, referring back to the discussion in Sec. III, one only needs to retain the completely symmetric contractions of repeated fields. Because each entry of the flavor tensor in front of a term is evaluated separately, the computational time of this approach increases sharply with the number of flavors. Concerning derivatives, BASISGEN deals with the integration-by-parts redundancies in a way which is different than the one described in this work; however, it is likely that the two methods are equivalent.

\section{Information beyond the number of operators}

It is possible to track each field flavor separately, both with the traditional method described here (see also [43]) and with the Hilbert series method (this leads to a so-called multigraded Hilbert series). However, doing so is very time consuming; therefore in the following I will analyze the information which can be extracted from both methods when the field flavor indices are not expanded.

The Hilbert series approach counts operators of a certain type for an arbitrary number of generations $n$, telling us, for example, that there are $n(n+1) / 2$ operators of the type $L L H H$. At first sight, the traditional method might seem to yield more information: it computes that the $n \times n$ flavor matrix $\kappa_{i j}$ in $\kappa_{i j} L_{i} L_{j} H H$ is symmetric; i.e., it transforms under the irreducible representation $\square=\{2\}$ of $S_{2}$. This implies that there are $\mathcal{S}(\{2\}, n)=n(n+1) / 2$ operators of this type, so the second result (the permutation symmetry) implies the first one (the operator counting). But does it contain more information? For this particular example, the answer is no: there is no extra information in the permutation symmetry because from the number of operators as a function of $n$ it follows that $\kappa$ is necessarily a symmetric matrix.

More generally, we can frame the discussion as follows. The traditional method provides the symmetry of the parameter tensors as a sum $\sum_{\lambda \vdash m} r_{\lambda} \lambda$ of irreducible representations $\lambda$ of some permutation group $S_{m}$, each with multiplicity $r_{\lambda}$. From here one can always calculate the total number of operators as a function of the number of flavors $n$,

$$
\text { \#operators }(n)=\sum_{\lambda \vdash m} r_{\lambda} \mathcal{S}(\lambda, n),
$$

while the Hilbert series method provides only the function \#operators $(n) .{ }^{21}$ Knowing the left-hand side of this expression, is it possible to retrieve the integer coefficients $r_{\lambda}$ ? In simple cases it is. The quantity $\mathcal{S}(\lambda, n)$ is a polynomial function of $n$ of degree $m$ (because $\lambda$ is a representation of $S_{m}$, hence it is a partition of $m$ ), with no constant term, which we can express as

$$
\mathcal{S}(\lambda, n) \equiv \sum_{i=1}^{m} S_{\lambda i} n^{i}
$$

We can obtain the $r_{\lambda}$ coefficients from \#operators $(n)$ in Eq. (91) if and only if all the polynomials $\mathcal{S}(\lambda, n)$ for different partitions $\lambda$ of $m$ are linearly independent. In turn, this is the same as requiring that all rows of the $S$ matrix above are linearly independent. With either two or three repeated fields ( $m=2$ or 3 ), the expressions

\footnotetext{
${ }^{21}$ The number of flavors $n$ should be seen as a variable here; otherwise \#operators $(n)$ becomes just a number and some information given by the Hilbert series method is lost.
} 


$$
\begin{gathered}
\mathcal{S}(\{2\}, n)=\frac{n(n+1)}{2}, \\
\mathcal{S}(\{1,1\}, n)=\frac{n(n-1)}{2}, \\
\mathcal{S}(\{3\}, n)=\frac{(n+2)(n+1) n}{6}, \\
\mathcal{S}(\{2,1\}, n)=\frac{(n+1) n(n-1)}{3}, \\
\mathcal{S}(\{1,1,1\}, n)=\frac{n(n-1)(n-2)}{6},
\end{gathered}
$$

imply that Eq. (91) is reversible, since the matrices

$$
S=\left(\begin{array}{cc}
\frac{1}{2} & \frac{1}{2} \\
-\frac{1}{2} & \frac{1}{2}
\end{array}\right)_{m=2},\left(\begin{array}{ccc}
\frac{1}{3} & \frac{1}{2} & \frac{1}{6} \\
-\frac{1}{3} & 0 & \frac{1}{3} \\
\frac{1}{3} & -\frac{1}{2} & \frac{1}{6}
\end{array}\right)_{m=3}
$$

are invertible. Therefore, whenever a field appears repeated at most 3 times in an interaction, the Hilbert series method provides the same information as the traditional method. But for $m>3$ the rows of matrix $S$ are never linearly independent: this can be seen just by counting its columns $(m)$ and its rows [equal in number to the distinct ways $p(m)$ of partitioning $m$ ]. The function $p(m)$ (called the partition function) becomes bigger than $m$ starting at $m=4$, increasing almost exponentially for very large $m$, so for four or more fields, the traditional method does provide some extra information which is not obtainable with the Hilbert series method described in the literature. For example, there is one linear relation among the $\mathcal{S}(\lambda, n)$ for $m=4$, namely

$$
\begin{aligned}
& \mathcal{S}(\{3,1\}, n)+\mathcal{S}(\{2,1,1\}, n)=3 \mathcal{S}(\{2,2\}, n) \\
& \quad\left[=n^{2}\left(n^{2}-1\right) / 4\right] .
\end{aligned}
$$

Therefore, from the fact that there are $n^{2}\left(n^{2}-1\right) / 4$ operators of type $d^{c} d^{c} d^{c} d^{c}\left(d^{c}\right)^{*}\left(e^{c}\right)^{*}$ in SMEFT it is impossible to infer what is the $S_{4}$ symmetry of the four $d^{c}$ 's; it might be $3\{2,2\}$ or $\{3,1\}+\{2,1,1\}$ (it turns out to be the latter case, so one Lagrangian term is enough to write down all these operators). This is the smallest SMEFT operator where the issue arises, and it has dimension nine, so it is fair to say that the extra information given by the traditional method is either minimal or nonexistent at all, unless the dimension of the operators is fairly large.

\section{Counting terms with derivatives}

Another important point worth mentioning is that the permutation symmetry of operators with derivatives is unclear, due to the integration-by-part redundancies.
Consider the example in Table III with the operators of the kind $\partial^{4} S^{4}$, where $S$ is a scalar field which does not change under gauge transformations. There are operators with symmetries $S_{2} \times S_{2}, S_{2} \times S_{1} \times S_{1}$, and $S_{4}$ depending on where the derivatives are applied (these are the operators with $0 \mathcal{D}$ 's in the table), and one must cross out some of them due to redundancies which have other permutation symmetries (they are associated with the operators with one or more $\mathcal{D}$ 's). It is hard to access what is the overall permutation symmetry of what remains after this subtraction. In fact, just as with the Hilbert series method, it is not even clear if any of the three types of operators- $S S\left(\partial^{2} S\right)\left(\partial^{2} S\right)$, $S(\partial S)(\partial S)\left(\partial^{2} S\right)$, and $(\partial S)(\partial S)(\partial S)(\partial S)$ —an be completely crossed out; hence one cannot tell how to distribute the four derivatives over the four $S$ scalars. At least not without extra considerations.

In light of this complication, one might simply compute the total number of operators and ignore all other information since one cannot make good use of it. However, there is perhaps a better alternative where less information is thrown away. For simplicity, consider the operators with only two derivatives and $m$ scalar singlets $S$ : there are $(\partial S)^{2} S^{m-2}$ operators, with a symmetry $\{2\}_{(\partial S)} \times\{m-2\}_{(S)}$, and redundancies $\mathcal{D}\left[(\partial S) S^{m-1}\right]$ with a symmetry $\{1\}_{(\partial S)} \times$ $\{m-1\}_{(S)}$. So, we need an $m$-index parameter tensor $\kappa$ to multiply/contract with the $(\partial S)^{2} S^{m-2}$ operators, and it must have a $\{2\} \times\{m-2\}$ permutation symmetry, with those components transforming as $\{1\} \times\{m-1\}$ removed. The number of nonredundant operators must be

$$
\begin{aligned}
& \mathcal{S}(\{m-2\}, n) \mathcal{S}(\{2\}, n)-\mathcal{S}(\{m-1\}, n) \mathcal{S}(\{1\}, n) \\
& \quad= \begin{cases}0 & \text { if } n=1 \\
\frac{n(m-3)(m+n-3) !}{2(m-1) !(n-2) !} & \text { if } n>1\end{cases}
\end{aligned}
$$

However, we can go beyond this counting exercise and, roughly speaking, subtract the $\{1\} \times\{m-1\}$ representation of $S_{1} \times S_{m-1}$ from the $\{2\} \times\{m-2\}$ representation of $S_{2} \times S_{m-2}$. Notice that these two representations can be found inside the following $S_{m}$ representations exactly once in all cases:

$\{2\} \times\{m-2\} \subset\{m\}, \quad\{m-1,1\}, \quad$ and $\quad\{m-2,2\} ;$

$$
\{1\} \times\{m-1\} \subset\{m\} \quad \text { and } \quad\{m-1,1\} .
$$

Therefore, only the components of $\kappa$ transforming as $\{m-$ $2,2\}$ under $S_{m}$ permutations survive. ${ }^{22}$ And indeed it is

\footnotetext{
${ }^{22}$ Not all of them though: only those which transform as $\{2\} \times$ $\{m-2\}$ under $S_{2} \times S_{m-2}$ permutations. From the comments made in Sec. III one can infer that this corresponds exactly to 1 in $d(\{m-2,2\})=m(m-3) / 2$ components of $\kappa$ transforming as $\{m-2,2\}$ under $S_{m}$ permutations.
} 


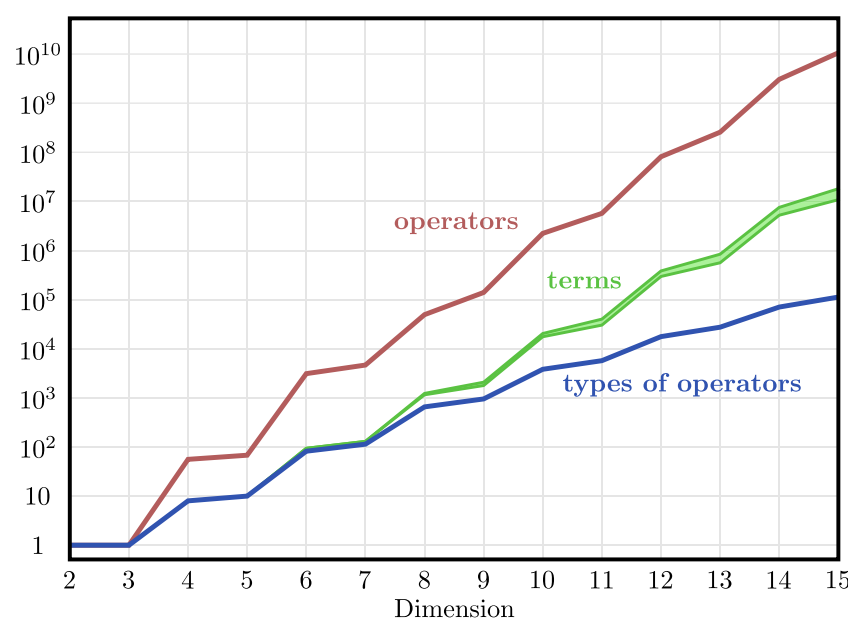

FIG. 2. Cumulative number of real operators, terms, and types of operators in SMEFT (as defined in Sec. II) up to a given dimension. Kinetics terms are ignored. Due to the presence of derivatives in some operators, the number of required Lagrangian terms cannot be determined exactly, but fairly stringent bounds (narrow green band) can be set on this quantity.

straightforward to check that the result in Eq. (100) is the same as $\mathcal{S}(\{m-2,2\}, n)$, using formula (32).

In this way, even in the presence of derivatives, it is still possible to obtain some permutation symmetry information of the operators with the redundancies removed. For example, in the more complicated case of operators of the kind $\partial^{4} S^{4}$ (see Table III) it turns out that the nonredundant operators are associated with the $S_{4}$ representation $\{4\}+\{2,2\}$, and indeed the total number of nonredundant operators in this case is

$\mathcal{S}(\{4\}, n)+\mathcal{S}(\{2,2\}, n)=\frac{1}{8} n\left(n^{3}+2 n^{2}+3 n+2\right)$.

Finally, it is worthwhile to discuss the number of terms (as defined in Sec. II) associated with operators with derivatives. In the absence of derivatives, the symmetry of the contraction of the fields can be expressed as a sum $\sum_{\lambda} r_{\lambda} \lambda$ of irreducible representations $\lambda$ of the relevant permutation group, with $r_{\lambda}$ representing the multiplicity of $\lambda$. We have seen already that these operators can be expressed with $t=\max \left(r_{\lambda}\right)$ terms, and no less.

Integration-by-parts redundancies complicate this calculation for operators with derivatives. Nevertheless, one can easily establish bounds on the minimal number of terms $t$ :

(i) $t$ cannot be smaller than the number of operators when considering only one generation of fields $(n=1)$.

\section{Operators}

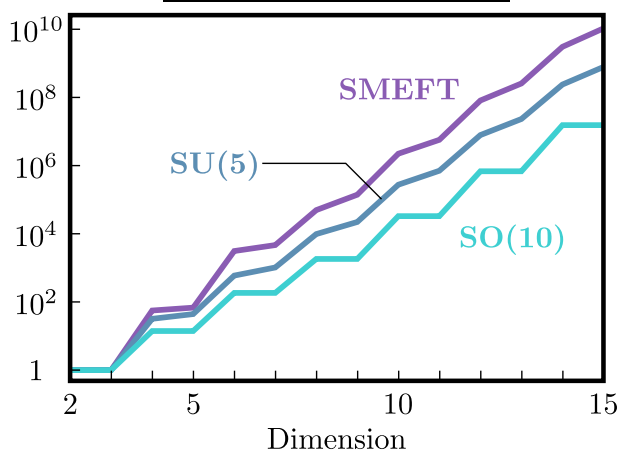

Terms

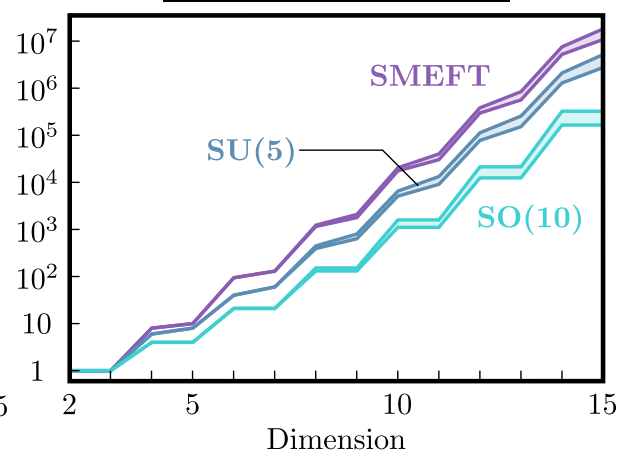

Types of operators

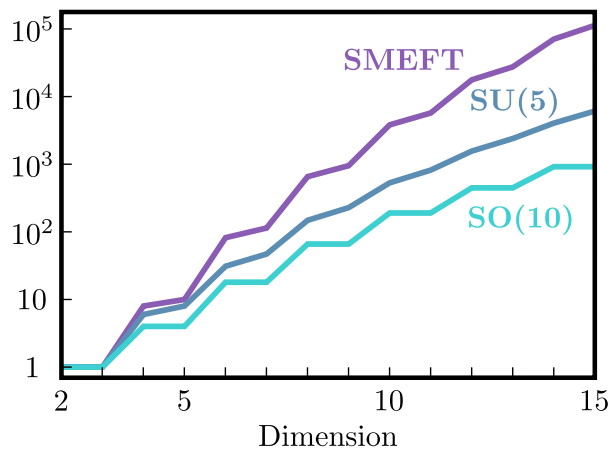

FIG. 3. Cumulative number of real operators, terms, and types of operators up to a given dimension in three models with different gauge symmetries - $S U(3) \times S U(2) \times U(1), S U(5)$, and $S O(10)$. The fermion and scalar content of each model is analogous (details can be found in the main text). As the symmetry group is enlarged, the number of operators, terms, and types of operators is reduced, as expected. 
(ii) $t$ does not need to be larger than the number of irreducible representations of the permutation group obtained after the procedure described a few paragraphs earlier.

(iii) $t$ also cannot exceed the number of terms needed to write all interactions if integration-by-parts redundancies are ignored.

For example, there is only one operator of the kind $\partial^{4} S^{4}$ if there is only one flavor of $S$, so $t \geq 1$. On the other hand, ignoring the redundancies in Table III, one could write all operators with three terms, so $t \leq 3$. Finally, the $\partial^{4} S^{4}$ interactions are associated with the $\{4\}+\{2,2\}$ permutation symmetry (two irreducible components), and therefore $t \leq 2$. In summary, these operators require either one or two Lagrangian terms $(1 \leq t \leq 2)$.

\section{E. Application to specific models}

The approach described in this work can be used to characterize individual interactions of a model. However, it would not be instructive to present here an exhaustive analysis of this kind. Instead, I will show some summary data of the interactions up to dimension 15 of three models: (a) the SMEFT; (b) an $S U(5)$ model with the left-handed fermion representations $3 \times \overline{\mathbf{5}}+3 \times \mathbf{1 0}$ and a scalar transforming as a 5; (c) an $S O(10)$ model with the left-handed fermion representations $3 \times \mathbf{1 6}$ and a real scalar transforming as a 10. These two latter models were picked to illustrate the effect of an enlarged symmetry group on the number of interactions, so the only fermion and scalar representations of $S U(5)$ and $S O(10)$ which were selected are those which contain the Standard Model fields.

The total number of real operators, terms, and types of operators up to dimension $d=2, \ldots, 15$ in these three models is represented graphically in Figs. 2 and 3. Exact numbers can found in Appendix. In the case of SMEFT, the number of operators up to dimension 15 , as well as the number of types of operators up to dimension 12 agree with the results obtained with the Hilbert series method in [18].

As mentioned earlier, the minimum number of terms which are needed to write down all operators can be calculated exactly for terms without derivatives. If there are derivatives, with the considerations made in this work it is only possible to derive bounds on this number. One can see from Figs. 2 and 3 that these bounds are fairly strict. Furthermore, the lower limit is close to (but never below) the number of operators in each of the models if they had a single fermion family.

\section{SUMMARY}

Operators in an effective field theory can be counted with the Hilbert series method. This technique, which requires the computation of some integrals, is very different from the one historically used in particle physics of simply multiplying together a model's fields in all possible ways, and retaining the combinations which are invariant under all relevant symmetries (such as the ones associated with the Lorentz and gauge groups). However, such a seemingly straightforward approach-which we may call the traditional method-is complicated by the fact that many operators have repeated fields, as well as derivatives.

This work describes a systematic and efficient way of addressing the problem of repeated fields. Derivatives can be handled with simple adaptations of the techniques known to work for the Hilbert series method. With these two difficulties solved, it becomes possible to count all operators of an effective field theory up to a high mass dimension, without relying on the Hilbert series technique.

The traditional method is potentially useful for various reasons. An obvious one is that it makes it possible to crosscheck results obtained in other ways. For example, the operators of the SMEFT were previously counted up to dimension 15 with the Hilbert series technique, but in the literature there was only confirmation of these numbers up to dimension eight. With the procedure described in this work, it is possible to verify the number of operators up to dimension 15, for an arbitrary number of fermion families.

Another interesting feature of the traditional method is that it provides more information beyond a simple operator counting. Among other things, the extra information can be used to count systematically the minimum number of Lagrangian terms required to encode a model's interactions. For instance, it indicates that all $Q Q Q L$ operators in SMEFT can be written down as a single term in the Lagrangian (in the past, this was not always clear), while the significantly more complicated interactions with twice as many fields, of the type $Q^{6} L^{2}$, need 11 terms.

The method described in this work was implemented in the program SYM2INT, which can readily be used to calculate the above operator properties in any field theory.

\section{ACKNOWLEDGMENTS}

I thank Xiaochuan Lu and Tom Melia for helping me understand one of their calculations in Ref. [18]. I acknowledge the financial support from the Grant Agency of the Czech Republic (GAČR) through Contract No. 17-04902S, from the Charles University Research Center UNCE/SCI/ 013, and from the Generalitat Valenciana through Grant No. SEJI/2018/033.

\section{APPENDIX: ADDITIONAL DATA}

The exact number of operators, terms, and types of operators for the three models described in Sec. V are given here in Tables IV-VI. Kinetic terms were not taken into account. Furthermore, note that any complex operator (plus its Hermitian conjugate) can be written as two real ones, and vice versa; the numbers in the tables below refer to real operators, terms, and term types. 
TABLE IV. Number of real operators, terms, and types of operators in SMEFT (as defined in Sec. II). Kinetics terms — of the kind $\partial \partial \phi \phi, \partial \psi^{*} \psi$, and $F F$ - are not taken into account. The number of operators up to dimension 15 shown here, as well as the number of types of operators up to dimension 12, agree with [18].

\begin{tabular}{lccc}
\hline \hline Dimension & \# operators & \# terms & \# types of operators \\
\hline 2 & 1 & 1 & 1 \\
3 & 0 & 0 & 0 \\
4 & 55 & 7 & 7 \\
5 & 12 & 2 & 2 \\
6 & 3045 & 84 & 72 \\
7 & 1542 & 36 & 32 \\
8 & 44807 & 1025 to 1102 & 541 \\
9 & 90456 & 628 to 852 & 296 \\
10 & 2092441 & 15769 to 18345 & 2868 \\
11 & 3472266 & 12726 to 19666 & 1898 \\
12 & 75577476 & 266031 to 343511 & 11942 \\
13 & 175373592 & 266802 to 457898 & 9824 \\
14 & 2795173575 & 4669533 to 6717444 & 43158 \\
15 & 7557369962 & 5599846 to 10567408 & 42206 \\
\hline \hline
\end{tabular}

TABLE V. Number of real operators, terms, and types of operators in an $S U(5)$ model with the left-handed fermion representations $3 \times \overline{\mathbf{5}}+3 \times \mathbf{1 0}$ and a scalar transforming as a $\mathbf{5}$.

\begin{tabular}{lccc}
\hline \hline Dimension & \# operators & \# terms & \# types of operators \\
\hline 2 & 1 & 1 & 1 \\
3 & 0 & 0 & 0 \\
4 & 31 & 5 & 5 \\
5 & 12 & 2 & 2 \\
6 & 549 & 32 & 23 \\
7 & 432 & 20 & 16 \\
8 & 8761 & 332 to 387 & 101 \\
9 & 12392 & 242 to 352 & 302 \\
10 & 252626 & 4459 to 5720 & 288 \\
11 & 431670 & 3990 to 6770 & 743 \\
12 & 7159650 & 68577 to 99666 & 834 \\
13 & 15425382 & 74252 to 142748 & 1651 \\
14 & 215670831 & 1139503 to 1850054 & 2056 \\
15 & 544121758 & 1449420 to 3067252 & \\
\hline \hline
\end{tabular}

TABLE VI. Number of real operators, terms, and types of operators in an $S O(10)$ model with the left-handed fermion representations $3 \times \mathbf{1 6}$ and a real scalar transforming as a $\mathbf{1 0}$. It can be shown that such a model does not have operators with an odd dimension.

\begin{tabular}{lccr}
\hline \hline Dimension & \# operators & \# terms & \# types of operators \\
\hline 2 & 1 & 1 & 1 \\
3 & 0 & 0 & 0 \\
4 & 13 & 3 & 3 \\
5 & 0 & 0 & 0 \\
6 & 170 & 17 & 14 \\
7 & 0 & 0 & 0 \\
8 & 1639 & 110 to 131 & 48 \\
9 & 0 & 0 & 0 \\
10 & 31059 & 977 to 1440 & 0 \\
11 & 0 & 0 & 257 \\
12 & 648654 & 0 & 0 \\
13 & 0 & 11319 to 19765 & 472 \\
14 & 14694065 & 0 & 0 \\
15 & 0 & 0 & to 302812 \\
\hline \hline
\end{tabular}


The reader will notice from Table VI that the $S O(10)$ model with left-handed fermions in the spinor representation $\mathbf{1 6}$ and a scalar in the vector representation $\mathbf{1 0}$ does not seem to have operators with an odd dimension. It can be shown analytically that this is indeed the case. Let us call $n_{16}, n_{16^{*}}, n_{10}$, and $n_{D}$ to the number of 16's, 16*'s, 10's, and derivatives $D$ in a given interaction (for all practical purposes, here $\mathcal{F}=D^{2}$ ). Then Lorentz invariance requires that $n_{16}+n_{D}$ and $n_{16^{*}}+n_{D}$ are both even numbers, while from the conjugacy classes of the $S O(10)$ representations we also conclude that $n_{16}+n_{16^{*}}$ must be even, and $2 n_{10}+$ $n_{16}-n_{16^{*}}$ must be a multiple of 4 . It follows that the operator dimension

$$
\begin{aligned}
d= & \frac{3}{2} n_{16}+\frac{3}{2} n_{16^{*}}+n_{10}+n_{D}=\left(n_{16}+n_{16^{*}}\right) \\
& +\left(n_{16^{*}}+n_{D}\right)+\frac{1}{2}\left(2 n_{10}+n_{16}-n_{16^{*}}\right)
\end{aligned}
$$

must be even.
[1] S. Weinberg, Baryon and Lepton Nonconserving Processes, Phys. Rev. Lett. 43, 1566 (1979).

[2] F. Wilczek and A. Zee, Operator Analysis of Nucleon Decay, Phys. Rev. Lett. 43, 1571 (1979).

[3] L. F. Abbott and M. B. Wise, The effective Hamiltonian for nucleon decay, Phys. Rev. D 22, 2208 (1980).

[4] C. J. C. Burges and H. J. Schnitzer, Virtual effects of excited quarks as probes of a possible new hadronic mass scale, Nucl. Phys. B228, 464 (1983).

[5] C. N. Leung, S. T. Love, and S. Rao, Low-energy manifestations of a new interaction scale: Operator analysis, Z. Phys. C 31, 433 (1986).

[6] W. Buchmüller and D. Wyler, Effective Lagrangian analysis of new interactions and flavor conservation, Nucl. Phys. B268, 621 (1986).

[7] B. Grzadkowski, M. Iskrzyński, M. Misiak, and J. Rosiek, Dimension-six terms in the Standard Model Lagrangian, J. High Energy Phys. 10 (2010) 085.

[8] R. Alonso, E. E. Jenkins, A. V. Manohar, and M. Trott, Renormalization group evolution of the Standard Model dimension six operators III: Gauge coupling dependence and phenomenology, J. High Energy Phys. 04 (2014) 159.

[9] L. Lehman, Extending the Standard Model effective field theory with the complete set of dimension-7 operators, Phys. Rev. D 90, 125023 (2014).

[10] Y. Liao and X.-D. Ma, Renormalization group evolution of dimension-seven baryon- and lepton-number-violating operators, J. High Energy Phys. 11 (2016) 043.

[11] Y. Liao and X.-D. Ma, Renormalization group evolution of dimension-seven operators in Standard Model effective field theory and relevant phenomenology, J. High Energy Phys. 03 (2019) 179.

[12] K. S. Babu and C. N. Leung, Classification of effective neutrino mass operators, Nucl. Phys. B619, 667 (2001).

[13] A. de Gouvêa and J. Jenkins, A survey of lepton number violation via effective operators, Phys. Rev. D 77, 013008 (2008).

[14] R. M. Fonseca and M. Hirsch, $\Delta L \geq 4$ lepton number violating processes, Phys. Rev. D 98, 015035 (2018).

[15] L. Lehman and A. Martin, Hilbert series for constructing Lagrangians: Expanding the phenomenologist's toolbox, Phys. Rev. D 91, 105014 (2015).
[16] L. Lehman and A. Martin, Low-derivative operators of the Standard Model effective field theory via Hilbert series methods, J. High Energy Phys. 02 (2016) 081.

[17] B. Henning, X. Lu, T. Melia, and H. Murayama, Hilbert series and operator bases with derivatives in effective field theories, Commun. Math. Phys. 347, 363 (2016).

[18] B. Henning, X. Lu, T. Melia, and H. Murayama, 2, 84, 30, 993, 560, 15456, 11962, 261485, ...: Higher dimension operators in the SM EFT, J. High Energy Phys. 08 (2017) 016; Erratum, J. High Energy Phys. 1909 (2019) 019.

[19] S. Benvenuti, B. Feng, A. Hanany, and Y.-H. He, Counting BPS operators in gauge theories: Quivers, syzygies and plethystics, J. High Energy Phys. 11 (2007) 050.

[20] B. Feng, A. Hanany, and Y.-H. He, Counting gauge invariants: The Plethystic program, J. High Energy Phys. 03 (2007) 090.

[21] J. Gray, Y.-H. He, A. Hanany, N. Mekareeya, and V. Jejjala, SQCD: A geometric apercu, J. High Energy Phys. 05 (2008) 099.

[22] E. E. Jenkins and A. V. Manohar, Algebraic structure of lepton and quark flavor invariants and $C P$ violation, J. High Energy Phys. 10 (2009) 094.

[23] A. Hanany, E. E. Jenkins, A. V. Manohar, and G. Torri, Hilbert series for flavor invariants of the Standard Model, J. High Energy Phys. 03 (2011) 096.

[24] A. V. Bednyakov, On three-loop RGE for the Higgs sector of 2HDM, J. High Energy Phys. 11 (2018) 154.

[25] A. Trautner, Systematic construction of basis invariants in the 2HDM, J. High Energy Phys. 05 (2019) 208.

[26] Anisha, S. Das Bakshi, J. Chakrabortty, and S. Prakash, Hilbert series and plethystics: Paving the path towards 2HDM- and MLRSM-EFT, J. High Energy Phys. 09 (2019) 035.

[27] R. M. Fonseca, Calculating the renormalisation group equations of a SUSY model with Susyno, Comput. Phys. Commun. 183, 2298 (2012).

[28] R. M. Fonseca, The Sym2Int program: Going from symmetries to interactions, J. Phys. Conf. Ser. 873, 012045 (2017).

[29] R. Slansky, Group theory for unified model building, Phys. Rep. 79, 1 (1981). 
[30] R. N. Cahn, Semi-Simple Lie Algebras and their Representations (Dover Publications, Mineola, 1985).

[31] T. P. Cheng and L. F. Li, Gauge Theory of Elementary Particle Physics (Oxford University Press, Oxford, 1984).

[32] W. Tung, Group Theory in Physics (World Scientific, Singapore, 1985).

[33] D. Bernstein, The computational complexity of rules for the character table of $S_{n}$, J. Symb. Comput. 37, 727 (2004).

[34] J. S. Frame, G. d. B. Robinson, and R. M. Thrall, The hook graphs of the symmetric group, Can. J. Math. 6, 316 (1954).

[35] R. P. Stanley, Enumerative Combinatorics: Volume 2 (Cambridge University Press, Cambridge, England, 1999).

[36] Y. Liao and X.-D. Ma, Operators up to dimension seven in Standard Model effective field theory extended with sterile neutrinos, Phys. Rev. D 96, 015012 (2017).
[37] B. Gripaios and D. Sutherland, DEFT: A program for operators in EFT, J. High Energy Phys. 01 (2019) 128.

[38] H. D. Politzer, Power corrections at short distances, Nucl. Phys. B172, 349 (1980).

[39] C. Arzt, Reduced effective Lagrangians, Phys. Lett. B 342, 189 (1995).

[40] B. Henning, X. Lu, T. Melia, and H. Murayama, Operator bases, $S$-matrices, and their partition functions, J. High Energy Phys. 10 (2017) 199.

[41] M. A. A. van Leeuwen, A. M. Cohen, and B. Lisser, LiE, A package for Lie group computations (1992), http://www .mathlabo.univ-poitiers.fr/ maavl/LiE/.

[42] R. M. Fonseca, GroupMath: A Mathematica package for group theory calculations (to be published), http:// renatofonseca.net/groupmath.php.

[43] J. C. Criado, BasisGen: Automatic generation of operator bases, Eur. Phys. J. C 79, 256 (2019). 